\title{
SAFE DESIGN OF COOLED TUBULAR REACTORS FOR EXOTHERMIC MULTIPLE REACTIONS: MULTIPLE- REACTION NETWORKS
}

\author{
E. J. WESTERINK and K. R. WESTERTERP \\ Chemical Reaction Engineering Laboratories, Department of Chemical Engineering, Twente University of \\ Technology, PO Rox 217, 7500 AE Enschede, The Netherlands
}

(Received 23 July 1986; accepted for publication 23 September 1987)

\begin{abstract}
A bstract-The model of the pseudo-homogeneous, one-dimensional cooled tubular reactor is applied to a multiple-reaction network. It is demonstrated for a network which consists of two parallel and two consecutive reactions. Three criteria are developed to obtain an integral yield which does not deviate more than a chosen fraction from the maximum yield that can be obtained in an isothermal reactor. The criteria enable us to choose relevant design and operating conditions for the safe execution of a reaction network in a tubular reactor. The method is illustrated for the production of maleic anhydride by air oxidation of benzene.
\end{abstract}

\section{INTRODUCTION}

Westerterp, Ptasinsky and Overtoom (Westerterp and Ptasinsky, 1984a, b; Westerterp et al., 1984a; Westerterp and Overtoom, 1985) discussed the design of cooled tubular reactors in which either two parallel or two consecutive reactions are carried out. An extensive literature survey is given by Westerterp and Ptasinsky (1984a). They demonstrated that, with a set of dimensionless groups exclusively characterizing either the reaction system or the design and operating conditions of the tubular reactor itself, criteria can be derived which relate uniquely the values of operating and design parameters with the required selectivity or integral vield and the reaction system parameters. Moreover they demonstrated that these criteria are much more strict than runaway criteria, so that the reactor always operates safely as long as the criteria are adhered to. These criteria were of the following form:

$$
U^{*}\left(\Theta_{\mathrm{ma}}-\Theta_{c}\right) / \Theta_{\mathrm{ad}}>=f\left(p, j_{P}, H, S_{P} \text { or } X_{P}\right) \text {. }
$$

The dimensionless group $U^{*}\left(\Theta_{\mathrm{ma}}-\Theta_{c}\right) / \Theta_{\mathrm{ad}}$ is representative for the ratio of the heat withdrawal rate and the maximum heat production rate; it only depends on the reactor design and operating variables. The other dimensionless group describing the reactor performance is $D a$, a dimensionless residence time in the reactor. At the right-hand side only properties of the reaction system (and not of the reactor) and the desired yield or selectivity appear. $\Theta_{\mathrm{ma}}$ as a maximum allowable temperature is a constraint which is governed by the desired yield or selectivity. The dimensionless groups describing the reaction system are $K, p, H$ and $j_{P}$.

Since the method works in the case of parallel as well as consecutive reactions, more complex systems combining both reaction types can be studied as well. In this study we will discuss the design and operation of a cooled tubular reactor for a combination of such reactions. To this end we have chosen the following network of first-order exothermic reactions, where $P$ is the desired product and the rate constants are of the
Arrhenius type:

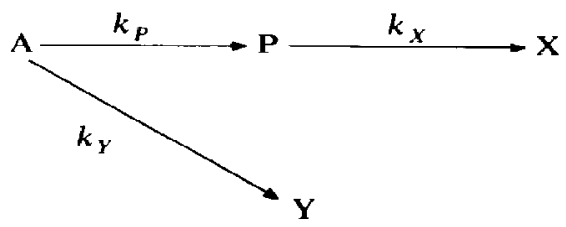

Since this reaction system consists of parallel and consecutive reactions it will behave like systems with either parallel or consecutive reactions, e.g. because of the consecutive reaction there will always be an optimal residence time and, because of the parallel reaction, the differential selectivity will always be less than one. In case only exothermic reactions are carried out the temperature will initially increase along the reactor tube, reach a maximum in the hot spot and afterwards decrease due to the heat exchange with the cooling medium. The optimal residence time or reactor length at a given reactor load depends on the shape of the temperature profile.

Westerterp and Westerink (1988) discussed the reaction system according to scheme (1) in case the reactions were carried out in a tank reactor. They discussed the relevant kinetic parameters and showed that the ratios of the activation energies, $E_{X} / E_{P}$ and $E_{Y} / E_{P}$, and of the pre-exponential factors, $A_{X} / A_{P}$ and $A_{y} / A_{P}$, are of major importance. We will restrict ourselves to systems where $A_{X}<A_{P}<A_{Y}$ and $E_{X}$ $<E_{P}<E_{Y}$ for the reasons outlined elsewhere (Westerterp and Westerink, 1988).

For the reaction system considered a possible Arrhenius plot is given in Fig. 1, where the logarithm of $k$ is plotted vs the reciprocal temperature. In general we desire to operate as far as possible at the left-hand side of this plot, because here temperatures and consequently reaction rates are high. At the onset of the reaction (no $P$ has been formed yet) we choose a temperature where the selectivity for the reactions $A \rightarrow$ $P$ and $A \rightarrow Y$ is high. For a certain minimum selectivity 


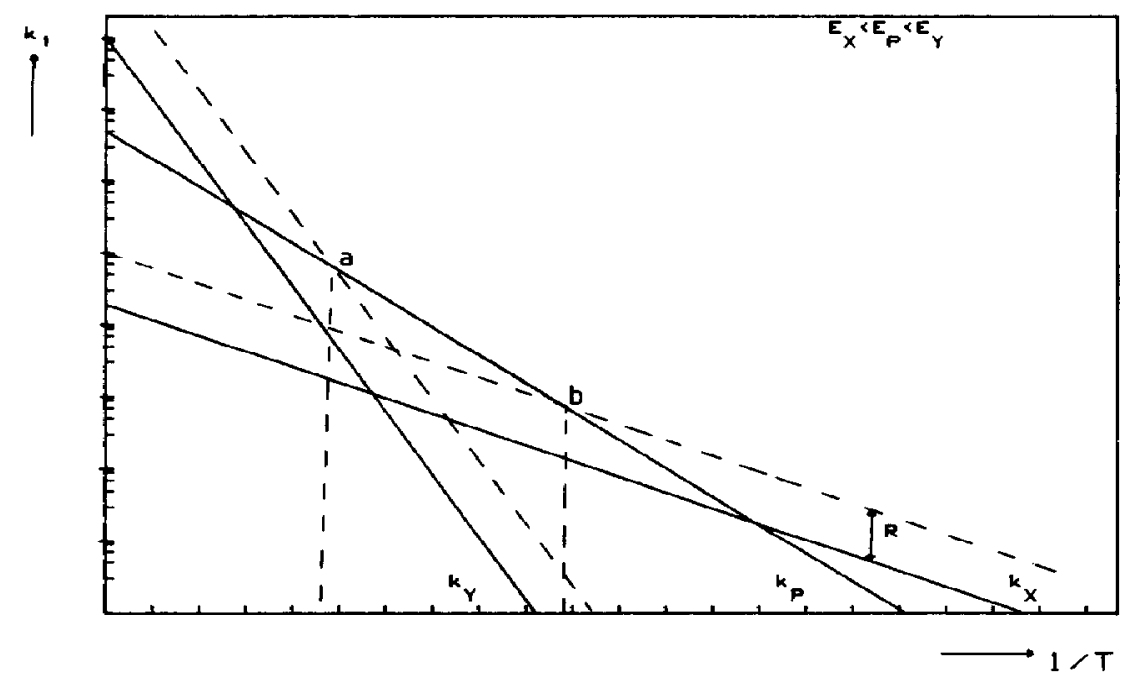

Fig. 1. General Arrhenius plot for the reaction system chosen [eq. (1)].

$S_{P}^{\prime}$ all reaction rates $k_{P}$ to the right of point $a$ are acceptable. As soon as some $P$ has been formed the consecutive reaction has also to be taken into account. For a certain yield of $X_{P}$ the ratio $k_{P} / k_{X}$ has to be larger than say a factor $R$. This implies that the reaction rates $k_{p}$ always have to be higher than indicated in Fig. 1 and that only reaction rates left of point $b$ are acceptable. In order to achieve the desired selectivity the reactor temperature $T$ must be in the range of $T_{b}<T<T_{a}$. We can understand that the higher our selectivity or yield requirements the smaller our allowable temperature range of $T_{b}-T_{a}$ will be.

The method of deriving the relevant dimensionless groups has been discussed by Westerterp et al. (Westerterp and Ptasinsky, 1984a, b; Westerterp et al., 1984a; Westerterp and Overtoom, 1985; Westerterp and Westerink, 1988) and will not be repeated here. These groups are based on a reference reaction rate constant $k_{R}$ taken at a reference temperature $T_{R}$. In our case we take this temperature, where both rate constants $k_{P}$ and $k_{Y}$ are equal; here $k_{R}$ and $T_{R}$ are true constants based on the particular reaction system only. The dimensionless groups $p, q, B, j_{P}, H_{X}$ and $H_{Y}$ are representative for the reaction system considered and $U^{*}, D a, \Theta_{\mathrm{ad}}, \Theta_{o}$ and $\Theta_{c}$ are representative for the operating and design variables. We refer to the Notation for their significance.

We must discriminate between reactor sections in which non-converted reactants can be recovered or not. In case recovery is possible the reactants are recycled to the inlet of the reactor. For our particular system in that case the reactor must operate at high selectivities and low conversions whereas the reactor design and operating conditions chosen are dominated by plant economics only. This case will not be discussed here (Westerterp and Ptasinsky, 1984a, b; Westerterp et al. 1984a; Westerterp and Overtoom, 1985; Westerterp and Westerink, 1988). In the case when no recovery is possible a maximum yield should be aimed at, hence we will discuss the design of a tubular reactor in which a certain minimum required yield has to be achieved. In the next sections we will introduce the basic equations that govern the conversion and temperature profiles in a cooled tubular reactor based on the pseudo-homogeneous onedimensional model. Next we will introduce a new type of conversion parameter that appears to be useful in case schemes with consecutive reactions are studied. Using this parameter selectivities in relation to isothermal and non-isothermal tubular reactors are discussed. Afterwards we will use the theory of isothermal reactors to formulate temperature regions for a nonisothermal tubular reactor that ensures a desired yield. Finally we will develop criteria that lead to the design of a tubular reactor in which this desired yield can be achieved. We will illustrate our method with an exampie.

\section{BASIC EQUATIONS}

In our tubular reactor the reactions of scheme (1) occur. In that reaction network $A$ is the reactant, $P$ the desired product and $X$ and $Y$ are undesired byproducts. All reactions are first-order and irreversible. The conversion rates are given by:

$$
\begin{aligned}
& R_{w A}=-\left(k_{P}+k_{Y}\right) C_{A} \\
& R_{w P}=k_{P} C_{A}-k_{X} C_{P} \\
& R_{w Y}=k_{Y} C_{A} \\
& R_{w X}=k_{X} C_{P} .
\end{aligned}
$$

Here $\boldsymbol{R}_{w J}$ is expressed in moles of species $J$ converted per unit time and per unit of catalyst mass.

We will use the pseudo-homogeneous, onedimensional model of the tubular reactor, the plugflow reactor, which has no radial temperature or 
concentration gradients. Moreover, we assume the temperature of the cooling medium to be constant along the tube length and the physical and chemical data $\rho_{g}, \rho_{b}, C_{\mathrm{pg}}$ and $-\Delta H$ to be independent of temperature. These assumptions lead to the following heat and mass balances for this type of reactor:

$$
\begin{aligned}
& u \frac{\mathrm{d} C_{A}}{\mathrm{~d} z}=R_{w A} \rho_{b} \\
& u \frac{\mathrm{d} C_{P}}{\mathrm{~d} z}=R_{w P} \rho_{b} \\
& u \frac{\mathrm{d} C_{Y}}{\mathrm{~d} z}=R_{w Y} \rho_{b} \\
& u \frac{\mathrm{d} C_{X}}{\mathrm{~d} z}=R_{w X} \rho_{b}
\end{aligned}
$$

$$
\begin{aligned}
\rho_{g} C_{\mathrm{pg}} u \frac{\mathrm{d} T}{\mathrm{~d} z}= & {\left[k_{P} C_{A}(-\Delta H)_{P}+k_{P} C_{A}(-\Delta H)_{Y}\right.} \\
& \left.+k_{X} C_{P}(-\Delta H)_{X}\right] \rho_{b}-\frac{4 U}{d_{t}}\left(T-T_{c}\right) .
\end{aligned}
$$

The reactor feed does not contain any $P, X$ and $Y$, so that $C_{P o}=C_{X o}=C_{Y o}=0$. We transform the species concentrations to conversions according to

$$
\begin{aligned}
\frac{C_{A o}-C_{A}}{C_{A o}} & =\frac{C_{P}}{C_{A o}}+\frac{C_{X}}{C_{A o}}+\frac{C_{Y}}{C_{A o}} \\
& =X_{A}=X_{P}+X_{X}+X_{Y}
\end{aligned}
$$

and make the equations dimensionless by multiplying by $L / C_{A o} u$ or $L / T_{R} u$ and introducing the dimensionless kinetic rate constants $K=k_{P} / k_{R}, K^{p}=k_{Y} / k_{K}$ and $B K^{q}=k_{X} / k_{R}$

$$
\begin{gathered}
\frac{\mathrm{d} X_{A}}{\mathrm{~d} Z}=D a\left(K+K^{p}\right)\left(1-X_{A}\right) \\
\frac{\mathrm{d} X_{P}}{\mathrm{~d} Z}=D a\left[K\left(1-X_{A}\right)-B K^{q} X_{P}\right] \\
\frac{\mathrm{d} X_{X}}{\mathrm{~d} Z}=D a B K^{q} X_{P} \\
\frac{\mathrm{d} X_{Y}}{\mathrm{~d} Z}=D a K^{p}\left(1-X_{A}\right) \\
\frac{\mathrm{d} \Theta \quad}{\mathrm{d} Z}=\operatorname{Da} \Theta_{\mathrm{ad}}\left[\left(K^{\prime}+H_{Y} K^{p}\right)\left(1-X_{A}\right)\right. \\
\left.+H_{X} B K^{q} X_{P}\right]-D a U^{*}\left(\Theta-\Theta_{c}\right) .
\end{gathered}
$$

Here the following dimensionless groups or variables are used:

$$
\begin{array}{cl}
U^{*}=\frac{4 U}{k_{R} d_{t} C_{\mathrm{pg}} \rho_{b} \rho_{g}} & D a=k_{R} \rho_{b} L / u \\
\Theta_{\mathrm{ad}}=\frac{(-\Delta H)_{P} C_{A o}}{T_{R} \rho_{q} C_{\mathrm{pg}}} & Z=z / L .
\end{array}
$$

Because of eq. (7) relations (8)-(12) are interrelated and this set of five can be reduced to a set of four equations by dividing each equation by $\mathrm{d} X_{A} / \mathrm{d} Z$ :

$$
\begin{gathered}
\frac{\mathrm{d} X_{P}}{\mathrm{~d} X_{A}}=\frac{K}{K+K^{p}}-\frac{B K^{q} X_{P}}{\left(K+K^{p}\right)\left(1-X_{A}\right)} \\
\frac{\mathrm{d} X_{X}}{\mathrm{~d} X_{A}}=\frac{B K^{q} X_{P}}{\left(K+K^{p}\right)\left(1-X_{A}\right)} \\
\frac{\mathrm{d} X_{Y}}{\mathrm{~d} X_{A}}=\frac{K}{\left(K+K^{p}\right)} \\
\frac{\mathrm{d} \Theta}{\mathrm{d} X_{A}}=\Theta_{\mathrm{ad}}\left[\frac{K+H_{Y^{p}}}{K+K^{p}}+\frac{H_{X^{B}} B K^{q} X_{P}}{\left(K+K^{p}\right)\left(1-X_{A}\right)}\right] \\
-\frac{U *\left(\Theta-\Theta_{c}\right)}{\left(K+K^{p}\right)\left(1-X_{A}\right)} .
\end{gathered}
$$

Because of the non-linearity of $K$ with respect to $\Theta$ being $K=\exp \left[j_{P}(1-1 / \Theta)\right]$ these differential equations cannot be solved analytically. Further the boundary conditions are:

$$
\begin{aligned}
Z=0 \quad T & =T_{o} \\
X_{A} & =0 \\
X_{P} & =0 \\
X_{X} & =0 \\
X_{Y} & =0
\end{aligned}
$$

\section{CHOICE OF A CONVENIENT CONVERSION PARAMETER}

The term $X_{P} /\left(1-X_{A}\right)$ appears in the mass balances for $P$ and $X$ as well as in the differential equation describing the reactor temperature profiles. Later on we will discuss the differential selectivity in both isothermal and non-isothermal reactors. Since the differential selectivity $S_{P}^{\prime}=\mathrm{d} X_{P} / \mathrm{d} X_{A}$ is given by eq. (13) it is convenient to use the term $X_{P} /\left(1-X_{A}\right)$ instead of $X_{A}$. Since $X_{A}$ increases along the reactor length, the term $X_{P} /\left(1-X_{A}\right)$ must increase along the reactor length to be a suitable replacement for $X_{A}$, so that

$$
\frac{\mathrm{d}\left[X_{P} /\left(1-X_{A}\right)\right]}{\mathrm{d} Z}>0 .
$$

Since also $\mathrm{d} X_{A} / \mathrm{d} Z>0$, the following criterion holds too:

$$
\frac{\mathrm{d}\left[X_{P} /\left(1-X_{A}\right)\right]}{\mathrm{d} \boldsymbol{X}_{A}}>0 .
$$

Working out the left-hand side of this equation leads with $\mathrm{d} X_{P} / \mathrm{d} X_{A}=S_{P}^{\prime}$ to

$$
\frac{\mathrm{d}\left[X_{P} /\left(1-X_{A}\right)\right]}{\mathrm{d} X_{A}}=\left[S_{P}^{\prime}+X_{P} /\left(1-X_{A}\right)\right] /\left(1-X_{A}\right) \text {. }
$$

We have to realize that in view of the consecutive character of the reaction $A \rightarrow P \rightarrow X$ we have to stop the reaction as soon as $\mathrm{d} X_{P} / \mathrm{d} X_{A}=S_{P}^{\prime}=0$, otherwise we pass the point of the maximum yield of $P$. In our region of interest of $S_{P}^{\prime}>=0$ the right-hand side of eq. 
(17) is positive, so that $X_{P} /\left(1-X_{A}\right)$ increases continuously. The term $X_{P} /\left(1-X_{A}\right)$ can be used as long as it increases with increasing $X_{A}$. Beyond the maximum of $X_{P}$ the value of $S_{P}$ is negative because there is a net consumption of $P$. Still eq. (17) can be used up till $-S_{P}^{\prime}$ has become equal to $X_{P} /\left(1-X_{A}\right)$.

\section{CUR VES OF CONSTANT DIFFERENTIAL SELECTIVITY}

The differential selectivity $S_{P}^{\prime}$ as described by eq. (13) can be studied in a two-dimensional plot of $\Theta$ vs $X_{p} /(1$ $-X_{A}$ ) with $S_{P}^{r}$ as a parameter. Equation (13) can be rewritten as

$$
X_{P} /\left(1-X_{A}\right)=\frac{K-\left(K+K^{P}\right) S_{P}^{\prime}}{B K^{q}}
$$

Differential isoselectivity curves of $\Theta$ vs $X_{P} /\left(1-X_{A}\right)$ are plotted in Fig. 2 for several values of $S_{P}^{\prime}$. Apparently for each $S_{P}^{\prime}(>0)$ value a maximum value of $X_{P} /\left(1-X_{A}\right)$ exists. In the previous paragraph we showed that $X_{P} /\left(1-X_{A}\right)$ increases with increasing $X_{A}$ as long as $S_{P}>0$, so at the maximum value of $X_{P} /(1$ $\left.-X_{A}\right)$ the conversion $X_{A}$ has also reached its highest value for a constant value of $S_{P}^{\prime}$. The yield of $P$ is related to the conversion of $A$ by multiplying the latter by the integral selectivity or in the case of constant differential selectivity by the differential selectivity $S_{P}^{\prime}$. Hence for a given differential selectivity the maximum yield is achieved at the maximum of $X_{P} /\left(1-X_{A}\right)$. Using these maxima it is possible to derive an optimal temperature profile. Starting at low temperatures and high selectivities the profile passes through the maxima: this profile will lead to the absolute maximum yield since for each differential selectivity the maximum contribution to the integral yield is obtained. As can be concluded from this optimal profile it is not possible to achieve a yield arbitrarily close to $100 \%$ as was possible in case of either only parallel or only consecutive reactions (Westerterp and Ptasinsky, 1984a, b; Westerterp et al., 1984a; Westerterp and Overtoom, 1985). Also this optimal temperature profile cannot be achieved in practice since in that case the optimum reactor temperature increases from $0 \mathrm{~K}$ up to infinity. We have to realize that in Fig. 2 the vertical scale covers a range of $0.4 T_{R}-1.6 T_{R}$, whereas $T_{R}$ usually has values of $400-1000 \mathrm{~K}$. In this plot $T_{R}$ $=848 \mathrm{~K}$. Even a part of this optimum profile is hard to approach because also runaway has to be prevented. In practice because of the prevention of runaway the reactor will operate in a rather narrow temperature range, almost isothermally. Hence, instead of optimal temperature profiles optimal isothermal reactors should be studied.

If the inlet temperature $\Theta_{\circ}$ equals the temperature of the cooling medium $\Theta_{c}$ and the highest temperature $\Theta_{\text {hs }}$ is reached in the hot spot, the reactor will operate in between the isotherms $\Theta_{c}$ and $\Theta_{\mathrm{hs}}$.

\section{THE BEHAVIOUR OF ISOTHERMAL REACTORS}

The behaviour of isothermal reactors is described by eqs (13)-(15). In practice we are interested in the desired product $P$ only, so that solving eq. (13) for a constant value of $K$ gives us

$$
X_{\text {Piso }}=\frac{\left(1-X_{A i s o}\right)^{B K^{q} /\left(K+K^{p}\right)}-\left(1-X_{\text {Aiso }}\right)}{1+K^{p-1}-B K^{q-1}} .
$$

With increasing conversions $X_{\text {Aiso }}$ the yield $X_{P \text { iso }}$ will initially increase, reach a maximum at $X_{P \max }$ and beyond this maximum the yield will decrease. This is shown in Fig. 3, where $X_{P \text { iso }}$ is plotted vs $X_{\text {Aiso }}$ for a given set of kinetic parameters and for several temperatures. The maximum yield is reached when the differential selectivity has just become zero. Hence, using

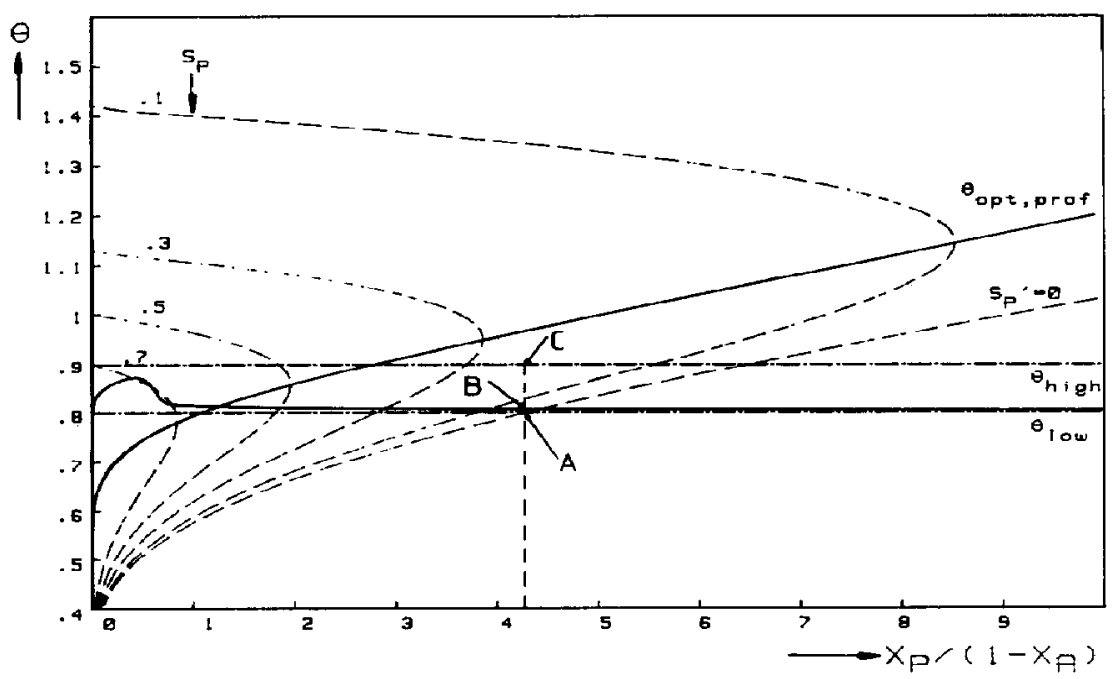

Fig. 2. Lines of constant differential selectivity and the optimal temperature profile. Data are $p=1.5, q$ $=0.8, B=0.11, j_{P}=15$. 


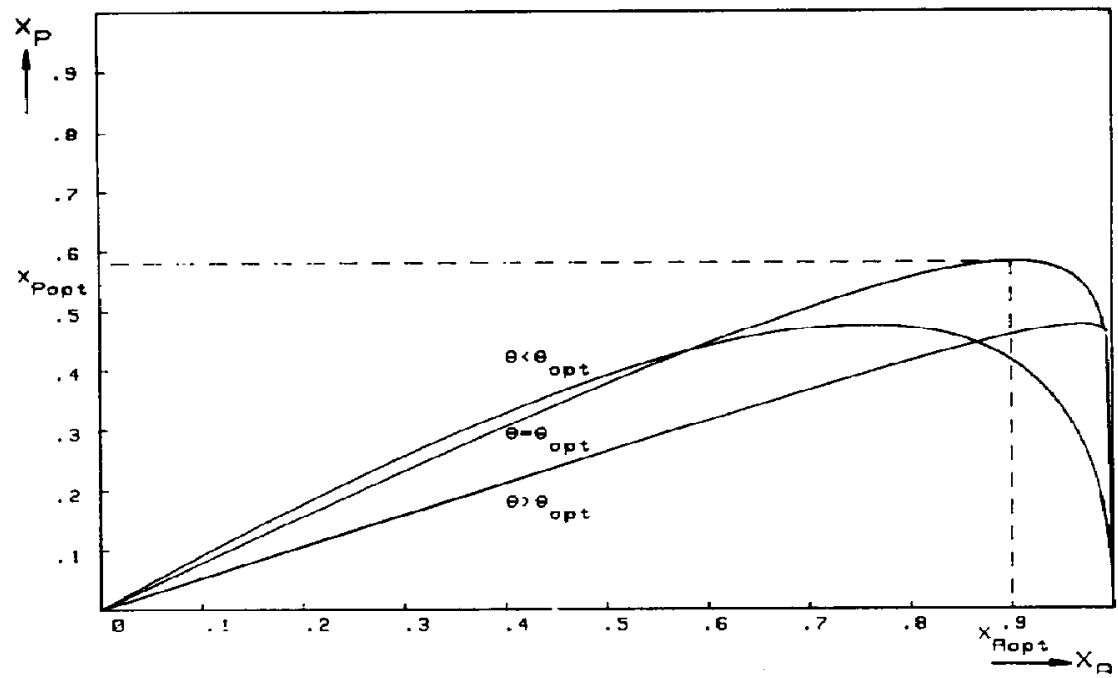

Fig. 3. Yield vs conversion in an isothermally operating reactor, at optimal isothermal conditions and at a temperature above and below $\Theta_{\text {opt }}$.

eq. (13):

$$
\left(1-X_{A \max }\right)=B K^{q-1} X_{P \max }
$$

Substitution into eq. (19) leads to

$$
X_{P \max }=\frac{K}{B K^{q}}\left(\frac{K+K^{p}}{B K^{q}}\right) \frac{1}{\frac{B K^{p}}{K+K^{p}-1}}
$$

and

$$
X_{A \max }=1-\left(\frac{K+K^{p}}{B K^{q}}\right)^{\frac{1}{B+K^{q}}} .
$$

The maximum yield $X_{P_{\max }}$ still depends on the temperature. Setting the derivative of eq. (21) with respect to $\Theta$ equal to zero gives the following implicit equation to determine the optimal temperature at which the highest maximum yield $\boldsymbol{X}_{\text {Popt }}$ of all maximum yields $X_{P_{\max }}$ is achieved in an isothermal tubular reactor:

$$
\begin{aligned}
\left(1-\frac{K+K^{p}}{B K^{q}}\right)^{2} & +\left[1-\frac{K+K^{p}}{B K^{q}}+\ln \left(\frac{K+K^{p}}{B K^{q}}\right)\right] \\
& \times\left(\frac{K}{B K^{q}}+\frac{p-q}{1-q} \frac{K^{p}}{B K^{q}}\right)=0 .
\end{aligned}
$$

In Fig. 3 the isotherm with $\Theta=\Theta_{\text {opt }}$ gives the highest maximum yield that can be achieved in an isothermally operating reactor. Figure 4 is a cross-plot of Fig. 3 .

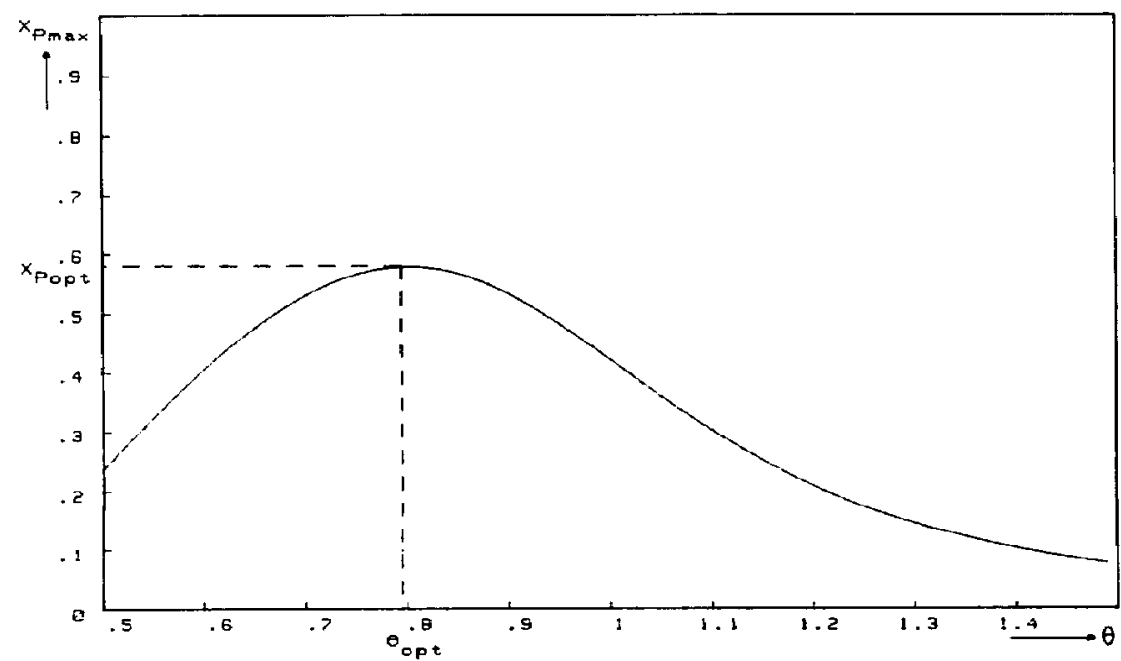

Fig. 4. Maximum and optimal yield in an isothermally operating reactor as a function of the reactor temperature. Same data as for Fig. 2. 
Here the maximum achievable yield $X_{P \max }$ under isothermal conditions is given as a function of $\Theta$, the maximum in this curve is the optimal yield $X_{\text {Popt }}$.

For a given temperature the required residence time $D a_{\max }$ to reach the conversion $X_{A \max }$ is given by eq. (8), which after integration gives

$$
D a_{\max }=\frac{-\ln \left(1-X_{A \max }\right)}{K+K^{p}} .
$$

At this residence time the reaction has to be stopped because otherwise there would be a net consumption of the desired product $\mathbf{P}$.

6. THE BEHAVIOUR OF NON-ISOTHERMAL REACTORS

The trajectory in a non-isothermal reactor is given by eqs (13) and (16). In case exothermic reactions are carried out the temperature will increase until the hot spot is reached, afterwards the temperature will gradually decrease. For multiple reactions one may expect more than one hot spot. In case of two consecutive reactions a first hot spot may occur due to the first reaction $A \rightarrow P$, a second may occur due to the consecutive reaction $P \rightarrow X$. In the Appendix it is proven that no multiple hot spots will occur in our case. Hence the trajectory has only one maximum.

The temperature in the hot spot is found by setting eq. (16) equal to zero:

$$
\begin{aligned}
\Theta_{\mathrm{ad}}\left[K+H_{Y} K^{p}+\right. & \left.H_{X} B K^{9} X_{P} /\left(1-X_{A}\right)\right] \\
& -U^{*}\left(\Theta-\Theta_{c}\right) /\left(1-X_{A}\right)=0 .
\end{aligned}
$$

Using the relation $X_{P}=S_{P h s} X_{A}$, we find that

$$
X_{A h s}=\frac{1-\frac{U^{*}\left(\Theta_{\mathrm{hs}}-\Theta_{c}\right)}{\Theta_{\mathrm{ad}}\left(K_{\mathrm{hs}}+H_{Y} K_{\mathrm{hs}}^{p}\right)}}{1-\frac{H_{X} B K_{\mathrm{hs}}^{q} S_{P h s}}{K_{\mathrm{hs}}+H_{Y} K_{\mathrm{hs}}^{p}}}
$$

or also

$$
\begin{aligned}
& X_{P} /\left(1-X_{A}\right)_{\mathrm{hs}} \\
& =\frac{U^{*}\left(\Theta_{\mathrm{hs}}-\Theta_{c}\right) / \Theta_{\mathrm{ad}}-K_{\mathrm{hs}}-H_{Y} K_{\mathrm{hs}}^{p}}{H_{X} B K_{\mathrm{hs}}^{q}-\frac{U^{*}\left(\Theta_{\mathrm{hs}}-\Theta_{c}\right)}{\Theta_{\mathrm{ad}} S_{\mathrm{Phs}}}}
\end{aligned}
$$

In these equations $S_{P h s}$ is the integral selectivity as achieved up to the hot spot. The integral selectivity will be a mean value of the achieved differential selectivities at the reactor inlet $\left(X_{A}=0\right)$ and at the hot spot $\left(X_{A}\right.$ $=X_{\text {Ahs }}$ ). The differential selectivity is given by eq. (13); this relation will be used to estimate the integral selectivity in the hot spot. We will discuss several possibilities to estimate $S_{P h s}$. Firstly we may substitute the differential selectivity at inlet conditions $\left(X_{A}=0\right.$, $\Theta=\Theta_{o}$ ), which is, according to eq. (13):

$$
S_{P_{1}}=\frac{1}{1+K_{o}^{P}} \text {. }
$$

The differential selectivity will always decrease in the first part of the reactor because of the increasing temperature and of the conversion of $P$. Hence, the integral selectivity in the first part of the reactor will be lower than $S_{P}^{\prime}\left(X_{A}=0\right)$, so that the influence of the undesired reactions is underestimated. In case of strongly exothermic undesired reactions $\left(H_{X}, I I_{Y}>1\right)$ using $S_{P}^{\prime}\left(X_{A}=0\right)$ will lead to a too low value for the hot spot temperature.

Secondly we can substitute the differential selectivity at the hot spot conditions:

$$
S_{P 2}=\frac{K_{\mathrm{hs}}}{K_{\mathrm{hs}}+K_{\mathrm{hs}}^{p}}-\frac{B K_{\mathrm{hs}}^{q}}{K_{\mathrm{hs}}+K_{\mathrm{hs}}^{P}} \frac{X_{P h s}}{1-X_{\text {Ahs }}} .
$$

In the hot spot both conversion and temperature are at their maximum values in the interval $0<=X_{A}<$ $=X_{\text {Ahs }}$, hence the differential selectivity is at a minimum value. Consequently we overestimate the heat production by the undesired reactions. Using $S_{P 2}$ in the case when $H_{X}$ or $H_{Y}>1$ leads to values for $\Theta_{h s}$ that are far too high.

As a third alternative, giving values of $S_{P h s}$ between $S_{P}^{\prime}\left(X_{A}=0\right)$ and $S_{P}^{\prime}\left(X_{A}=X_{A \mathrm{hs}}\right)$, we studied

$$
S_{P 3}=\frac{1}{1+K_{\mathrm{hs}}^{p-1}} .
$$

We can easily verify that $S_{P 2}<S_{P 3}<S_{P 1}$.

There remains the question of which value of $S_{P}$ to use in eq. (26). In general we are only interested in reaction networks that give a high yield. In that case the consecutive reaction is still largely suppressed in the first part of the reactor tube (Westerterp and Overtuom, 1985), hence before the hot spot almost no undesired product $X$ is formed. Consequently the conditions in the hot spot are mainly dominated by the heat effects of the desired and the undesired parallel reactions only. In previous articles (Westerterp and Ptasinsky, 1984a, b; Westerterp et al., 1984a; Westerterp and Overtoom, 1985; Westerterp and Westerink, 1988) we demonstrated that a certain maximum allowable temperature should not be exceeded in order to achieve a desired yield or selectivity. We will apply the same method so a too high estimate for the hot spot temperature should be selected which means that the initial heat production should be overestimated in order to be on the safe side. Consequently it depends on the heat of reaction of the undesired parallel reaction whether we must over- or underestimate the selectivity.

From numerical evaluation it appeared that $S_{p_{3}}$ gave the most accurate predictions. The conversion due to the parallel reaction was slightly overestimated, hence $S_{P 3}$ will be used only in the case when $H_{Y}>1$. By doing so we are sure that we are on the safe side and that reactor temperatures will be lower than assumed. $S_{P 1}$ will be used in case $H_{Y}<1$. Using $S_{P 2}$ leads to far too high values of the hot spot temperature in the case when $H_{Y}>1$. This leads to the instructions:

$$
\begin{gathered}
\text { - use } S_{P_{1}} \text { if } H_{Y}<1 \\
\text { - use } S_{P_{3}} \text { if } H_{Y}>1
\end{gathered}
$$

Substituting either $S_{P 1}$ or $S_{P 3}$ for $S_{P h s}$ into eq. (26) or 
(27) enables us to calculate the locus of maxima curves in a $\Theta_{\mathrm{hs}}-X_{\mathrm{Ahs}}$ or a $\Theta_{\mathrm{hs}}-X_{\text {phs }} /\left(1-X_{\text {Ahs }}\right)$ plot. Several locus curves are shown in Fig. 5 , in which also some trajectories are plotted for several values of $U^{*}, \Theta_{c}$ and $\Theta_{\text {ad }}$. As can be seen, the slope of the trajectory outside the locus of maxima curve is positive while the slope is negative in the area enveloped by the locus of maxima curve.

The locus of maxima curve is influenced by the reaction parameters $j_{P}, p, q, H_{X}, H_{Y}$ and $B$, and by two design and operating parameters, $\Theta_{c}$ and $U^{*} / \Theta_{\text {ad }}$ Figure 5(a) and (b) demonstrate that at increasing ratio $U * / \Theta_{\mathrm{ad}}$ or at decreasing $\Theta_{\mathrm{c}}$ the locus curves shift to the left. At certain combinations of values of $\Theta_{c}$ and
$U * / \Theta_{\text {ad }}$, the locus curves start to intersect the $X_{A}=0$ axis, as was the case for two parallel or two consecutive reactions. Two points of intersection are possible, the corresponding temperatures $\Theta_{i 1}$ and $\Theta_{i v}$ are indicated. In the case when the reactor is operated at an inlet temperature $\Theta_{a}$ which is lower than $\Theta_{\mathrm{i}}$, the reactor temperature will initially increase until the maximum temperature is reached on the lower branch of the locus curve; afterwards the temperature will decrease and eventually approach $\Theta_{c}$. In case the inlet temperature is chosen between $\Theta_{i 1}$ and $\Theta_{i u}$ the reactor temperature will decrease along the entire length of the reactor tube. As long as $\Theta_{o}<\Theta_{i v}$ no runaway will occur.

In the case when the locus of maxima curve does not
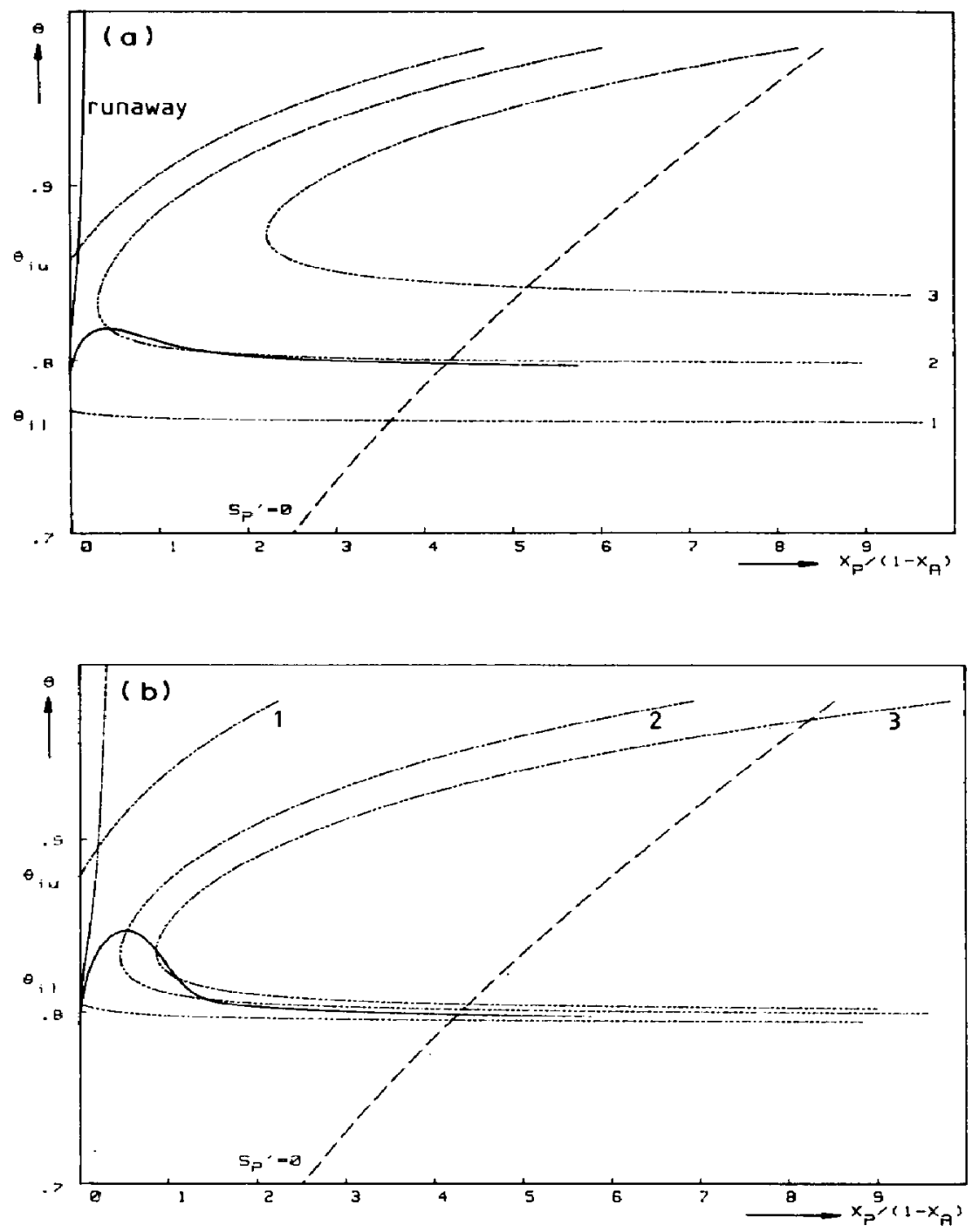

Fig. 5. Locus of maxima curves. In (a) $\Theta_{c}$ is varied $(0.76,0.79$ and 0.82$)$, and in (b) $U *$ is varied $(0.60,0.30$ and 0.25 ). Two temperature trajectories are plotted in both figures, one under runaway conditions (trajectory $b$, $\Theta_{\mathrm{ad}}=1.2$ ) and one under stable conditions (trajectory $\mathrm{a}, \Theta_{\mathrm{ad}}=0.6$ ). Data are $p=1.5, q=0.8, B=0.11, j_{P}$ $=15, H_{X}=H_{Y}=2, U^{*} / \Theta_{\text {ad }}=1.3[(a)]$ and $\Theta_{c}=0.79[(b)]$. The trajectories refer to the locus curve with $\Theta_{c}$ $=0.79$ and $U^{*}=0.30$, respectively. 
intersect the $X_{A}=0$ axis runaway is possible for any value of $\Theta_{0}$ : whether runaway occurs or not depends on the values of $U^{*}$ and $\Theta_{\mathrm{ad}}$. This is demonstrated in Fig. 5.

\section{A CRITERION WHICH GUARANTEES A DESIRED YIELD}

Until now we studied the possible yield in isothermal reactors and the temperature behaviour of non-isothermal reactors. Furthermore in the Appendix we proved that only one hot spot occurs in the case of our system of multiple exothermic reactions.

From Fig. 4 we can see that there is only one isothermal reactor temperature $\Theta_{\text {opt }}$ at which the maximum of the maximum yield is achieved. We called this the optimum yield $X_{P o p t}$; for yields in isothermal reactors, lower than the optimum, two isotherms are possible to achieve equal maximum yield, one at a temperature higher and one at a temperature lower than $\Theta_{\text {opt }}$.

In practice a reactor will not operate isothermally, so that the maximum isothermal yield cannot be obtained. The selectivity achieved is worse than the selectivity achieved under isothermal conditions due to the temperature profile in the non-isothermal reactor. In the case when the non-isothermal reactor operates in a temperature range below $\Theta_{\text {opt }}$ the optimal conversion, where the reaction has to be stopped and achieved at $S_{P}^{\prime \prime}=0$, is lower than $X_{A o p t}$; on the other hand at a temperature above $\Theta_{\text {opt }}$ the optimal conversion is higher than $X_{\text {fopt }}$, as can be seen in Fig. 2.

We will now compare a non-isothermal reactor with two isothermal reactors. We assume that one isothermal reactor operates at $\Theta_{\text {low }}$ and the other one at $\Theta_{\text {high }}$; furthermore we assume that the non-isothermal reactor operates above $\Theta_{\text {low }}=\Theta_{c}=\Theta_{o}$ and that for the hot spot temperature holds $\Theta_{\mathrm{hs}}=<\Theta_{\text {high }}$. In the non-isothermal reactor the temperature will initially increase and if $U^{*}$ is sufficiently high the hot spot temperature $\Theta_{\mathrm{hs}}$ will remain below $\Theta_{\text {high }}$. Beyond the hot spot the temperature decreases and, for long reactors, will become almost equal to $\Theta_{\text {low }}$ at the outlet of the reactor. In both the non-isothermal reactor and the isothermal one the reaction has to be stopped as soon as $S_{p}^{\prime}=0$. For the isothermal reactor the reaction has to be stopped at point $A$ in Fig. 2. At point $A$ $X_{P \text { iso }} /\left(1-X_{\text {Aiso }}\right)$ for the isothermal reactor is equal to $X_{p} /\left(1-X_{A}\right)$ at point $B$, which corresponds to the nonisothermal reactor. For the non-isothermal reactor the conversion $X_{A}$ at point $B$ will be higher because of the higher average temperature level. As a consequence $X_{P_{\text {non-iso }}}<X_{\text {Piso }}$ for $X_{P} /\left(1-X_{A}\right)=X_{\text {Piso }} /\left(1-X_{A \text { iso }}\right)$. Therefore we may conclude that a non-isothermal reactor that operates as indicated in Fig. 2 between two temperatures $\Theta_{\text {low }}$ and $\Theta_{\text {high }}$ will not necessarily have a yield that is higher than $X_{P \max }\left(\Theta_{\text {low }}\right)$, not even if $\Theta_{\text {high }}$ $=\Theta_{\mathrm{opt}}$. This is a very important phenomenon which will be accounted for in the determination of our design criteria.

Now assume that we aim for a certain yield $X_{P d}$. This yield is defined as the yield which does not deviate more than a chosen fraction $d$ from the maximum obtainable yield $X_{P o p t}$. Hence

$$
X_{P d}>=(1-d) X_{P o p t} .
$$

We state that there are two temperatures $\Theta_{\text {mi }}$, being the lowest required temperature, and $\Theta_{\text {ma }}$, being the maximum allowable temperature, between which the reactor should operate in order to achieve a desired yield $X_{P d}$. In the ultimate situation $\Theta_{\mathrm{mi}}$ will equal $\Theta_{\mathrm{ma}}$ and the reactor has to be operated isothermally in order to achieve the desired yield.

We will use Figs 2 and 6 to define the lower $\left(\Theta_{\mathrm{mi}}\right)$ and upper $\left(\Theta_{\mathrm{ma}}\right)$ temperature limit in our tubular reactor. In order to operate at a high temperature level we assume that the minimum temperature $\Theta_{\mathrm{mi}}$ is equal to

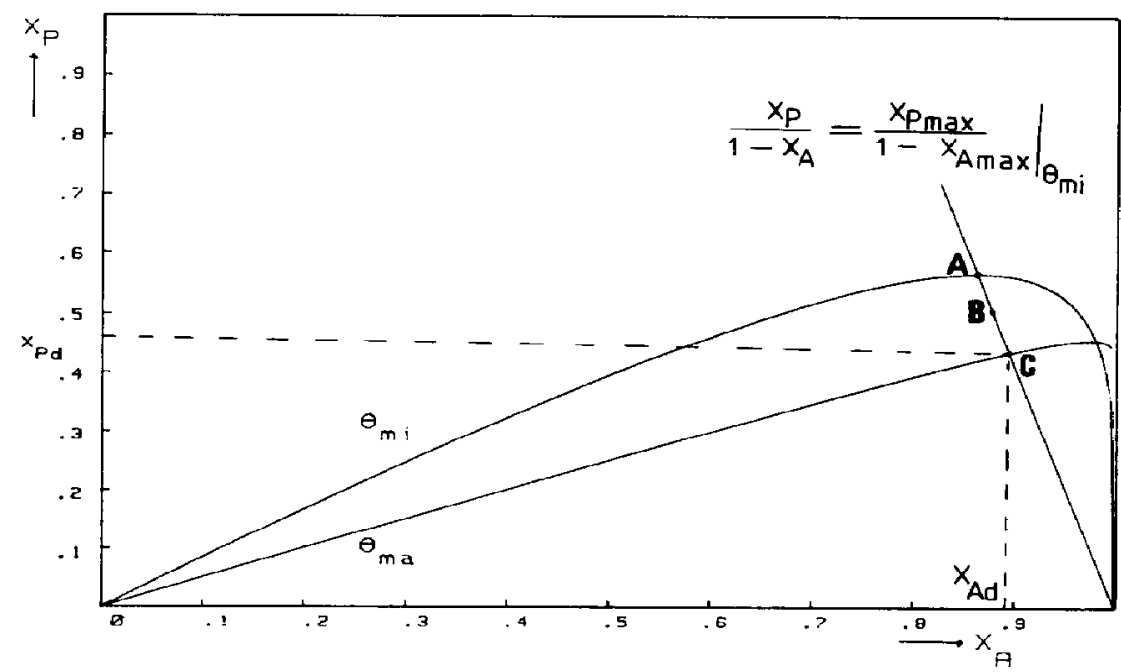

Fig. 6. Determination of the maximum allowable temperature $\Theta_{\text {ma }}$ and the minimum required temperature $\Theta_{\mathrm{mi}}$ 
or higher than the optimal temperature $\Theta_{\text {opt }}$; meanwhile $\Theta_{\mathrm{mi}}$ should be chosen in such a way that $X_{P \max }\left(\Theta_{\mathrm{mi}}\right)$ is higher or equal to the desired yield $X_{P d}$ since the yield achieved under non-isothermal conditions is always lower than the yield achieved under isothermal conditions. From Fig. 2 we can understand that for a constant value of $X_{P} /\left(1-X_{A}\right)$ the yield achieved at point $B$ will be lower than the yield achieved at point $A$ because of the higher conversion of $A$ at point $B$, where point $A$ is the conversion $X_{p} /(1$ $\left.-X_{A}\right)$ at which the lower isotherm reaches its maximum yield $X_{P_{\max }}\left(\Theta_{\operatorname{mi}}\right)$. In Fig. 6 both isotherms $\Theta_{\mathrm{mi}}$ and $\Theta_{m a}$ are plotted as well as the line for a constant value of $X_{P} /\left(1-X_{A}\right)$ equal to $X_{P \text { iso }} /\left(1-X_{A \text { iso }}\right)$ as given by point $A$. Therefore this line passes through the maximum of the isotherm at $\Theta_{\mathrm{mj}}$. The line intersects the isotherm $\Theta$ ma before the isotherm $\Theta_{\mathrm{ma}}$ has reached its maximum. We can easily see that the yield achieved in the non-isothermal reactor $\left(X_{\text {Pnon-iso }}\right)$ is always higher than the yield achieved at conversion $X_{A}=X_{A d}$ by the isothermal reactor. Now for each value of $\Theta_{\mathrm{mi}}$, with $\Theta_{\text {opt }}<\Theta_{\mathrm{mi}}<\Theta\left(X_{P d}\right)$, a corresponding temperature $\Theta_{\text {ma }}$ can be calculated in such a way that two conditions are met simultaneously:

(a) $X_{P} /\left.\left(1-X_{A}\right)\right|_{\theta_{\mathrm{mi}}}=X_{P} /\left.\left(1-X_{A}\right)\right|_{\Theta_{\mathrm{ma}}}$

and

(b) $\left.X_{P}\left(\Theta_{\mathrm{ma}}\right)\right|_{X_{P}\left(\Theta_{\mathrm{mi}}\right) /\left[1-X_{A}\left(\Theta_{\mathrm{mi}}\right)\right]}=X_{P d}$.

So, as long as the reactor operates between $\Theta_{\mathrm{mi}}$ and $\Theta_{\text {ma }}$, the maximum yield will always be higher than $\boldsymbol{X}_{\boldsymbol{P d} \text {. }}$

Since the reactor must operate between $\Theta_{\mathrm{mi}}$ and $\Theta_{\mathrm{ma}}$ the following demands can be formulated:

Since the reactor should operate above the minimum temperature $\Theta_{\mathrm{mi}}$ the lowest temperature, being either the inlet temperature $\Theta_{o}$ or the coolant temperature $\Theta_{c}$, should be higher than $\Theta_{\mathrm{mi}}$. Therefore

$$
\Theta_{\text {o }} \text { and } \Theta_{c}>=\Theta_{\mathrm{mi}} \text {. }
$$

The highest temperature is reached in the hot spot of the reactor, where the upper limit $\Theta_{\text {ma }}$ must not be exceeded. This leads to the following condition for the hot spot temperature:

$$
\boldsymbol{\Theta}_{\mathrm{hs}}<=\boldsymbol{\Theta}_{\mathrm{ma}} \text {. }
$$

These limits are calculated in the following procedure. First we calculate the optimum temperature $\Theta_{\text {opt }}$ using eq. (23) and the isotherm $\Theta_{\max }$ at which the desired yield $\boldsymbol{X}_{P d}$ would have been achieved if the reactor was operated isothermally. We use eq. (21) and solve it for the temperature: two solutions are possible the highest one is used as $\Theta_{\text {max }}$. Now we select a minimum required temperature $\Theta_{\mathrm{mi}}$ in such a way that

$$
\Theta_{\text {opt }}<\Theta_{\text {mi }}<\Theta_{\text {max }}
$$

Now we calculate the maximum isothermal yield, using eq. (21), that can be achieved in a reactor operating at $\Theta=\Theta_{\text {mi }}$. Next we calculate the conversion achieved at this point using eq. (22). Now construct the line for constant value of $X_{P} /\left(1-X_{A}\right)$ with the yield and conversion calculated above. We intersect this line with the curve for isothermal yields in a reactor operating at $\Theta_{\mathrm{ma}}$ using eq. (19). The following values for $X_{P \text { iso }}$ and $X_{A \text { iso }}$ are used:

$$
X_{P \text { iso }}=X_{P d}
$$

and

$$
X_{P} /\left.\left(1-X_{A}\right)\right|_{\Theta_{\mathrm{mi}}}=X_{P i s o} /\left(1-X_{A i s o}\right) .
$$

Equation (19) is solved for $\Theta_{\mathrm{ma}}$, and we use this temperature for the upper limit in our temperature region.

Using these limits we will derive design criteria for the tubular reactor that guarantec a required minimum yield.

\section{THE FIRST CRITERION}

In the previous paragraph it was shown that the reactor must operate between $\Theta_{\mathrm{mi}}$ and $\Theta_{\mathrm{ma}}$. The minimum temperature $\Theta_{\mathrm{mi}}$ sets a lower limit to the coolant temperature $\Theta_{c}$. We now will use the locus of maxima curves to formulate criteria for the design and operating variables that limit the hot spot temperature in such a way that it remains below $\Theta_{\mathrm{ma}}$.

The first criterion is derived from the locus curves intersecting the $X_{A}=0\left[\right.$ or $\left.X_{P} /\left(1-X_{A}\right)=0\right]$ axis. We demonstrated that for $\Theta_{0}<=\Theta_{i l}$ the reactor temperature is always below $\Theta_{\mathrm{il}}$ and for $\Theta_{\mathrm{il}}<=\Theta_{o}<\Theta_{\mathrm{iu}}$ the reactor temperature is always below $\Theta_{o}$. Hence, the highest possible reactor temperature is either $\Theta_{i 1}$ or $\Theta_{i u}$. Both values are found by setting $X_{A}$ equal to zero in eq. (26):

$$
\frac{U^{*}\left(\Theta-\Theta_{c}\right)}{\Theta_{\mathrm{ad}}}=K+H_{Y} K^{p}
$$

Substitution of $\Theta_{\mathrm{ma}}$ into this equation leads to our first criterion:

$$
\frac{U *\left(\Theta_{\mathrm{ma}}-\Theta_{c}\right)}{\Theta_{\mathrm{ad}}}=>K_{\mathrm{ma}}+H_{Y} K_{\mathrm{ma}}^{p} .
$$

As can be concluded from eq. (33) our first criterion does not depend on the integral selectivity in the hot spot.

A special case is given by the locus curve that has only one point of intersection with the $X_{A}=0$ axis. For a given value of $\Theta_{c}$, as can be seen in Fig. 5(a) and (b) there is one value of $U^{*} / \Theta_{\text {ad }}$ for which $\Theta_{\mathrm{il}}$ and $\Theta_{\mathrm{iu}}$ coincide and where the locus curve just touches the $X_{A}$ $=0$ axis. Setting the derivative of eq. (25) with respect to $\Theta$ equal to zero defines the temperature $\Theta_{i}$ for which $\Theta_{i}=\Theta_{\mathrm{it}}=\Theta_{\mathrm{iu}}$ :

$$
\frac{K_{i}+H_{Y} K P}{K_{i}+p H_{Y} K \boldsymbol{P}}-\frac{j_{P}\left(\Theta_{i}-\Theta_{c}\right)}{T_{i}^{\overline{2}}}=0
$$

or in the case when $T_{i}-T_{c}$ is eliminated using eq. (32):

$$
\frac{U^{*}}{\Theta_{\mathrm{ad}}}-\frac{j_{P}\left(K_{i}+p H_{Y} K_{i}^{p}\right)}{\Theta_{i}^{2}}=0
$$

From eq. (34) we can see that either $\Theta_{c}$ or $\Theta_{i}$ can be 
chosen freely, so that for a given value of $\Theta_{c}$ the point of intersection $\left(\Theta_{i}\right)$ is fixed. Substitution of the maximum allowable temperature $\Theta_{\mathrm{ma}}$ into eq. (34) or (35) leads to a criterion that gives us the required coolant temperature or the required ratio $U^{*} / \Theta_{\text {ad }}$. It gives the lowest values of $U^{*} / \Theta_{\text {ad }}$ for which the first criterion is adhered to but it only can be applied if $\Theta_{c}$ according to eq. (34) is higher than $\Theta_{m i}$ because the minimum temperature in our reactor must be higher than $\Theta_{\mathrm{mi}}$ as was stated before.

Using eq. (33) or (34) as the criterion for the design always leads to stable reactors because the temperature can never rise above the point of intersection $\Theta_{i} l=$ $\Theta_{\mathrm{ma}}$ ). Also no estimated value of $S_{P}$ in the hot spot is required.

\section{THE SECOND CRITERION}

The second criterion is derived from locus curves that do not intersect the $X_{A}=0$ axis. The criterion is based on the property of trajectories for stable reactors that a trajectory starts at its maximum slope $\mathrm{d} \Theta / \mathrm{d} X_{A}$ at the inlet conditions: beyond the inlet the slope decreases due to the heat exchange with the cooling medium. Hence, our second criterion is based on the condition

$$
\left.\frac{\mathrm{d} \Theta}{\mathrm{d} X_{A}}\right|_{X_{A}=0}<=\left(\Theta_{\mathrm{ma}}-\Theta_{o}\right) / X_{A \mathrm{ma}}
$$

as illustrated in Fig. 7.

The slope $\mathrm{d} \Theta / \mathrm{d} X_{A}$ is given by eq. (16) and $X_{A \mathrm{ma}}$ by eq. (26), substituting $\Theta_{\text {ma }}$ for $\Theta_{\text {hs. }}$. The required value for $S_{P}$ can be determined using $S_{P 1}$ or $S_{P 3}$ as explained before. Substitution into condition (36) leads to the following expression for the second criterion:

$\Theta_{\mathrm{ad}}\left(\frac{K_{o}+H_{Y^{\prime}} K_{o}^{p}}{K_{o}+K_{o}^{p}}\right)-\frac{U^{*}\left(\Theta_{o}-\Theta_{c}\right)}{K_{o}+K_{o}^{p}}<=$

$$
\left(\Theta_{\mathrm{ma}}-\Theta_{o}\right)\left[\begin{array}{c}
1-\frac{H_{X} B K_{\mathrm{ma}}^{q} S_{P}}{K_{\mathrm{ma}}+K_{\mathrm{ma}}^{p}} \\
1-\frac{U^{*}\left(\Theta_{\mathrm{ma}}-\Theta_{c}\right)}{\Theta_{\mathrm{ad}}\left(K_{\mathrm{ma}}+H_{Y} K_{\mathrm{ma}}^{p}\right)}
\end{array}\right] .
$$

In the case when $\Theta_{o}$ equals $\Theta_{c}$ our second criterion simplifies to

$$
\begin{aligned}
& \frac{U^{*}}{\Theta_{\mathrm{ad}}}\left(\Theta_{\mathrm{ma}}-\Theta_{c}\right)>=\left(K_{\mathrm{ma}}+H_{Y} K_{\mathrm{ma}}^{p}\right) \\
& \times\left[\begin{array}{c}
\left(\Theta_{\mathrm{ma}}-\Theta_{c}\right)\left(1-\frac{H_{X} B K_{\mathrm{ma}}^{q} S_{p}}{K_{\mathrm{ma}}+H_{Y} K_{\mathrm{ma}}^{p}}\right) \\
\left.1-\frac{\Theta_{\mathrm{ad}}\left(\frac{K_{c}+H_{Y_{c}} K_{c}^{p}}{K_{c}+K_{\mathrm{c}}^{p}}\right)}{}\right]
\end{array}\right] .
\end{aligned}
$$

It can be concluded from a comparison of the two criteria (33) and (38) that they differ in the term

$$
1-\frac{\left(\Theta_{\mathrm{ma}}-\Theta_{c}\right)\left(1-\frac{H_{X} B K_{\mathrm{ma}}^{q} S_{P}}{K_{\mathrm{ma}}+H_{Y} K_{\mathrm{ma}}^{p}}\right)}{\Theta_{\mathrm{ad}}\left(\frac{K_{c}+H_{Y} K_{c}^{p}}{K_{c}+K_{c}^{p}}\right)}
$$

This term is smaller than one for high yields, so this criterion is a less stringent version of the first one. However, to apply this criterion the integral selectivity $S_{P}$ has to be estimated using either $S_{P 1}$ or $S_{P 3}$.

\section{THE THIRD CRITERION}

Assuming that no undesired product $X$ has been formed and that no heat is withdrawn before the hot spot, the temperature rise in the reactor is given by

$$
\begin{aligned}
\Theta_{\mathrm{hs}}-\Theta_{o} & =\Theta_{\mathrm{ad}}\left(X_{P h s}+H_{\gamma} X_{Y h s}\right) \\
& =\Theta_{\mathrm{ad}}\left[S_{P}+H_{Y}\left(1-S_{P}\right)\right] X_{\text {Ahs }}
\end{aligned}
$$

Setting $\Theta_{\mathrm{hs}}=\Theta_{\mathrm{ma}}$ and calculating $\left(X_{A}\right)_{\mathrm{ma}}$ with eq. (26)

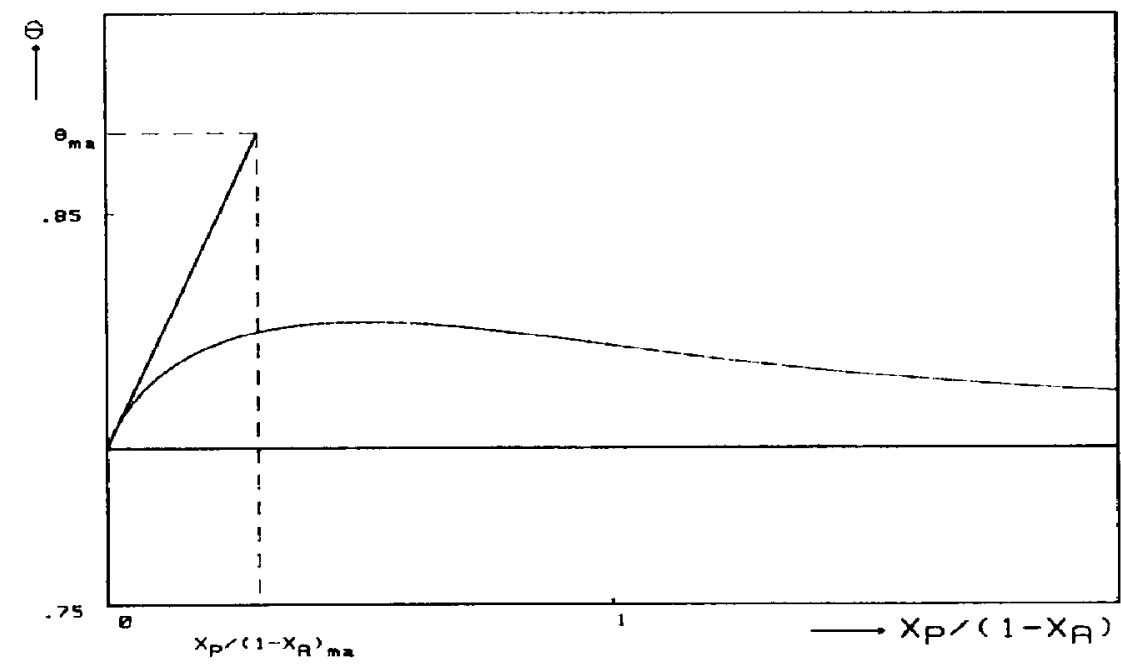

Fig. 7. Illustration of the second criterion (see text). 
and in the case when $\Theta_{o}$ equals $\Theta_{c}$, we find the following relation for the dimensionless number $U^{*} / \Theta_{\text {ad }}$ which is our third criterion:

$$
\begin{aligned}
\frac{U^{*}}{\Theta_{\mathrm{ad}}}= & \frac{K_{\mathrm{ma}}+H_{Y} K_{\mathrm{ma}}^{p}}{\Theta_{\mathrm{ma}}-\Theta_{c}} \\
& -\frac{K_{\mathrm{ma}}+H_{Y} K_{\mathrm{ma}}^{p}-S_{P} H_{X} B K_{\mathrm{ma}}^{p}}{\Theta_{\mathrm{ad}}\left[S_{P}+H_{Y}\left(1-S_{P}\right)\right]} .
\end{aligned}
$$

Intuitively it can be felt that the value $U^{*}$ must have a minimum; for small values of $\Theta_{\text {ma }}-\Theta_{c}$ the required value of $U^{*}$, despite the low reaction rates, must be large in order to keep $\Theta_{\mathrm{ma}}-\Theta_{c}$ at the required low value. On the other hand for large values of $\Theta_{\mathrm{ma}}-\Theta_{c}$ the local heat production rates near the hot spot are high, so $U^{*}$ should be high too. In between $U^{*}$ will reach a minimum. Hence, $U^{*}$ is a function of $\Theta_{m a}-\Theta_{c}$. Setting the derivative of $U^{*}$ with respect to $\Theta_{\mathrm{ma}}$ equal to zero leads to the formulation of the following implicit equation from which the optimum value of $\Theta_{c}$ can be calculated by trial and error:

$$
\begin{aligned}
\frac{K_{\mathrm{ma}}+H_{Y} K_{\mathrm{ma}}^{p}}{\left(\Theta_{\mathrm{ma}}-\Theta_{c}\right)^{2}}-\frac{j_{P}\left(K_{\mathrm{ma}}+p I I_{Y} K_{\mathrm{ma}}^{p}\right)}{\left(\Theta_{\mathrm{ma}}-\Theta_{c}\right) \Theta_{\mathrm{ma}}^{2}} \\
\quad+\frac{j_{P}\left(K_{\mathrm{ma}}+p H_{Y} K_{\mathrm{ma}}^{p}-S_{P} q I_{X} B K_{\mathrm{ma}}^{q}\right)}{\Theta_{\mathrm{ad}} \Theta_{\mathrm{ma}}^{2}\left[S_{P}+H_{Y}\left(1-S_{P}\right)\right]}=0 .
\end{aligned}
$$

For a given value of $\Theta_{\mathrm{ad}}$ and $\Theta_{\mathrm{ma}}$ the required value of $\Theta_{c}$ can be calculated; it should be checked if $\Theta_{c}$ lies within the region of acceptable values $\left(\Theta_{\mathrm{mi}}<\Theta_{c}\right.$ $<\Theta_{\text {ma }}$ ). If not, then the lowest possible value of $U^{*} / \Theta_{\mathrm{ad}}$ can be found by taking $\Theta_{\mathrm{c}}=\Theta_{\mathrm{mi}}$ and substituting $\Theta_{c}$ into eq. (39). Hence using eq. (40) the optimal value of $\Theta_{c}$ can be calculated which then can be substituted into eq. (39) to calculate the required value of $U^{*} / \Theta_{\text {ad }}$ according to the third criterion.

We must stress the fact that for the second and third criterion values of $S_{P}$ are required, which are calculated using either $S_{P 1}$ or $S_{P 3}$. These substitutions are valid only in case the heat effect of the consecutive reaction can be neglected before the hot spot is reached. If this is not the case, for instance if $H_{X}$ is considerably larger than 1 , only the first criterion is legitimate since for this criterion no estimates of $S_{P}$ are required.

\section{SELECTION OF PRACTICAL VALUES OF $\Theta$}

The required residence time is strongly dependent on the value of the coolant temperature. For isothermal reactors the required residence time $D a$ is calculated from eq. (24). For a non-isothermal reactor, as can be seen in Fig. 6 , the required yield $X_{P d}$ will be reached before $X_{A d}$. The longest residence time required to reach $X_{A d}$ is found if the isotherm $\Theta=\Theta_{m i}$ is followed. Since we select the coolant temperature $\Theta_{c}=\Theta_{\mathrm{mi}}$, the corresponding residence time $D a_{1 \mathrm{p}}$ for the isothermal reactor operating at the coolant temperature $\Theta_{c}$ is

$$
D u_{1 \mathrm{p}}=\frac{-1}{K_{c}+K_{c}^{p}} \ln \left(1-X_{A d}\right) .
$$

After setting a maximum for the tube length in the reactor to be built, that is a maximum allowable residence time, and with the known value of $\Theta_{\text {ma }}$ the value of $\Theta_{c}$ can be calculated. In practice the reactor operates at higher temperatures than $\Theta_{c}$ and the required yield will be reached before $X_{A d}$ so the actual residence time will be shorter than $D a_{1 p}$.

The shortest residence time in an isothermal reactor to reach the required yield is found for the highest possible temperature $\left(\Theta_{\mathrm{ma}}\right)$, which leads to

$$
D a_{\mathrm{sp}}=\frac{-1}{K_{\mathrm{ma}}+K_{\mathrm{ma}}^{p}} \ln \left(1-X_{A d}\right) .
$$

Equations (41) and (42) enable us to select reactor temperature regimes where reactor operation under realistic conditions is possible.

\section{DESIGN PROCEDURE FOR COOLED TUBULAR REACTORS}

In the previous paragraphs we discussed how a temperature region can be determined for which a tubular reactor achieves a minimum desired yield. We showed that the reactor should operate between two temperature limits, the lower limit $\Theta_{\mathrm{mi}}$ and the upper limit $\Theta_{m a}$. We stated that as long as $\Theta_{c}>=\Theta_{m i}$ and $\Theta_{h s}$ $<=\Theta_{m a}$ the reactor operates within this area. We also derived criteria that ensure that the hot spot temperature $\Theta_{\text {hs }}$ remains below a preset temperature $\Theta_{\text {ma }}$. Based on these results the following design procedure, leading to the design of a safely operating cooled tubular reactor for the execution of reaction networks, can be developed.

(1) Make an Arrhenius plot of all reactions involved and determine whether high or low temperatures or a region of temperatures are desired to obtain high yields. In case high temperatures are required the reactor should operate at the highest possible temperature, in case low temperatures are required the reactor should operate at the lowest possible temperature. These designs are dominated by plant economics and mechanical constraints. In the case when the reactor should operate inside a regime the following procedure can be used.

(2) For the reaction system considered determine the relevant reaction system parameters $k_{R}, T_{R}, p, q$, $B, j_{p}, H_{X}$ and $H_{Y}$.

(3) Calculate the absolute optimal temperature $\Theta_{\text {opt }}$ and the corresponding values of $X_{A o p t}$ and $X_{P o p t}$.

(4) Calculate for several values of $X_{P \text { iso }}<X_{P o p t}$ the isotherm $\Theta_{\max }$ from eq. (21) so that $X_{P \text { iso }}$ $=X_{P_{\max }}$. Also calculate the corresponding conversion $X_{A \max }$ with eq. (22) and the residence time $D a_{\max }$ with eq. (24). Now select a design value for the yield $X_{P d}$ that leads to an acceptable residence time $D a_{\max }$ under isothermal conditions.

(5) Calculate for the chosen value of $X_{P d}$ and for several values of $\Theta_{\text {mi }}$, where $\Theta_{\text {opt }}<\Theta_{\text {mi }}<\Theta_{\max }$, the maximum allowable temperature $\Theta_{\text {ma. }}$. Also 
calculate the corresponding conversion $X_{A d}$ and the longest possible residence time $D a_{1 \mathrm{p}}$. Now select temperature regimes that do not lead to too long residence times and also do not demand a too narrow temperature regime (so that the reactor would have to operate almost under isothermal conditions).

(6) Decide whether to use $S_{P 1}$ or $S_{P 3}$ and determine their values from eq. (28) or (30). Calculate for the remaining regimes as determined in step (5) the required values of $U^{*} / \Theta_{\text {ad }}$ using the three criteria for $\Theta_{\mathrm{ma}}$.

(7) Determine, for each value of $U^{*} / \Theta_{\text {ad }}$ and a range of reactor loads, the maximum tube diameter and the value of the heat transfer coefficient.

(8) Calculate the required optimal reactor length, using numerical integration techniques, for the configurations determined in step (6).

(9) Choose an economical design based mainly upon the required tube diameters, number of tubes and tube lengths.

(10) Check the design chosen for the effects of operational upsets, e.g. changes of the reactor load and of the feed concentration and if necessary correct the values chosen under (9).

\section{AN EXAMPLE}

In the previous sections we derived criteria that enable us to design a reactor that achieves a required yield. We will demonstrate the method by applying it to the industrial process of the oxidation of benzene with air to maleic anhydride.

One of the synthesis routes for the production of maleic anhydride is based on the direct air oxidation of benzene with a vanadium pentoxide catalyst. An excess of air is applied, but the reactant benzene cannot be recovered economically so the reactor must operate at high yields. The main by-products are $\mathrm{CO}_{2}$ and $\mathrm{H}_{2} \mathrm{O}$, which are formed according to

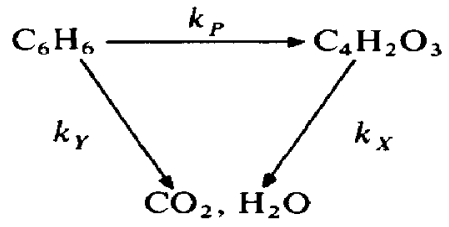

Kinetic data were presented by Wohlfahrt and Emig (1980) for the following system of reactions:

$$
\begin{aligned}
R_{w P} & =k_{P}\left[\mathrm{C}_{6} \mathrm{H}_{6}\right]-k_{X}\left[\mathrm{C}_{4} \mathrm{H}_{2} \mathrm{O}_{3}\right] \\
R_{w X} & =k_{X}\left[\mathrm{C}_{4} \mathrm{H}_{2} \mathrm{O}_{3}\right] .
\end{aligned}
$$

Here A stands for benzene, $P$ for the desired product maleic anhydride and $X$ and $Y$ are $\mathrm{CO}_{2}$ and $\mathrm{H}_{2} \mathrm{O}$ formed due to the consecutive and the parallel reactions, respectively. The corresponding rate constants are:

$$
\begin{aligned}
& k_{P}=4280 \exp \{-12,660 /[T(\mathrm{~K})]\} \mathrm{m}^{3} /(\mathrm{kg} \text { cat s) } \\
& k_{Y}=70,100 \exp \{-15,000 /[T(\mathrm{~K})]\} \mathrm{m}^{3} / \text { (kg cat s) } \\
& k_{X}=26 \exp \{-10,800 /[T(\mathrm{~K})]\} \mathrm{m}^{3} /(\mathbf{k g} \text { cat s) }
\end{aligned}
$$

Furthermore the following physical data are available:

$$
\begin{aligned}
C_{\mathrm{pg}} & =1.09 \mathrm{~kJ} /(\mathrm{kg} \mathrm{K}) \\
\rho_{g} & =1.01 \mathrm{~kg} / \mathrm{m}^{3} \\
\rho_{b} & =900 \mathrm{~kg} / \mathrm{m}^{3}
\end{aligned}
$$

and the concentration of benzene in the feed is

$$
C_{\text {Ao }}=0.83 \text { mole } \%
$$

which corresponds to a $\Theta_{\text {ad }}$ of 0.63 .

With these data the following parameters are calculated:

$$
\begin{array}{ccc}
T_{R}=848 \mathrm{~K} & & k_{R}=1.4 \times 10^{-3} \mathrm{~m}^{3} / \mathrm{kg} \mathrm{s} \\
j_{P}=14.9 & q=0.85 & H_{X}=0.77 \\
B=0.055 & p=1.18 & H_{Y}=1.77 \quad \Theta_{\mathrm{ad}}=0.63
\end{array}
$$

\section{DETERMINATION OF THE OPERATING AND DESIGN VARIABLES}

With eqs (21) (23) the absolute optimal yield $X_{P o p t}$ and the corresponding temperature $\Theta_{\text {opt }}$ and conversion $X_{\text {Aopt }}$ are calculated. The following results are obtained:

$$
\begin{aligned}
\Theta_{\mathrm{opt}} & =0.667 \\
X_{P \mathrm{opt}} & =0.590 \\
X_{\text {Aopt }} & =0.904 .
\end{aligned}
$$

Substituting these data into eq. (24) gives the required residence time:

$$
D a_{\mathrm{opt}}=k_{R} \rho_{b} L / u=1950
$$

which is far too high, requiring a residence time $L / u$ of $1550 \mathrm{~s}$. From these results we can conclude that a yield

$$
\begin{array}{ll}
\mathrm{C}_{6} \mathrm{H}_{6}+\frac{9}{2} \mathrm{O}_{2} \rightarrow \mathrm{C}_{4} \mathrm{H}_{2} \mathrm{O}_{3}+2 \mathrm{CO}_{2}+2 \mathrm{H}_{2} \mathrm{O} & (-\Delta H)_{P}=1850 \mathrm{~kJ} / \mathrm{mol} \\
\mathrm{C}_{6} \mathrm{H}_{6}+{ }_{2} 5 \mathrm{O}_{2} \rightarrow 6 \mathrm{CO}_{2}+3 \mathrm{H}_{2} \mathrm{O} & (-\Delta H)_{Y}=3274 \mathrm{~kJ} / \mathrm{mol} \\
\mathrm{C}_{4} \mathrm{H}_{2} \mathrm{O}_{3}+3 \mathrm{O}_{2} \rightarrow 4 \mathrm{CO}_{2}+\mathrm{H}_{2} \mathrm{O} & (-\Delta H)_{X}=1423 \mathrm{~kJ} / \mathrm{mol}
\end{array}
$$

In practice selectivities of $50-60 \%$ and benzene conversions of $85-95 \%$ are achieved, so yields of $40-50 \%$ are realistic. The following conversion rates are given:

$$
\begin{aligned}
-R_{w A} & =k_{P}\left[\mathrm{C}_{6} \mathrm{H}_{6}\right]+k_{Y}\left[\mathrm{C}_{6} \mathrm{H}_{6}\right] \\
R_{w Y} & =k_{Y}\left[\mathrm{C}_{6} \mathrm{H}_{6}\right]
\end{aligned}
$$

lower than $59 \%$ has to be accepted in order to obtain a realistic reactor design. For several isothermal maximum yields $X_{P_{\max }}$ the required temperatures $\Theta_{\max }$ were calculated. The required residence time $D a_{\max }$ 
is calculated with eq. (24). The results are listed in Table 1.

As can be seen from Table 1 yields close to the optimal yield $X_{P o p t}=0.59$ require extremely long residence times. We will accept residence times between 1 and $10 \mathrm{~s}$ and therefore aim for a yield of $48 \%$ which can be achieved in an isothermal reactor operating at a temperature $\Theta_{\max }=0.94$. In Table 2 the maximum allowable temperature $T_{\mathrm{ma}}$ is determined for several minimum temperatures $T_{\mathrm{mi}}$. Also the maximum allowable temperature rise is given as well as the longest possible residence time $D a_{\mathrm{lp}}=D a\left(X_{A d}, T_{\mathrm{mi}}\right)$ to reach the yield $X_{P d}$. It can be seen that in case of minimum temperatures close to the maximum allowable temperature the maximum residence times are relatively short but an almost isothermal operating reactor is required, which will lead to high values of $U^{*}$ and uneconomical reactor designs. On the other hand in case of large allowable regions the minimum temperature is low and therefore the required resi-

Table 1. Temperatures, conversion and residence time for several yields in isothermal reactors. $j_{P}=14.9, B=0.055$, $p=1.18, q=0.85$

\begin{tabular}{lllll}
\hline$X_{P}$ & $\Theta_{\max }$ & $X_{A \max }$ & $D a_{\max }$ & $\tau(\mathrm{s})$ \\
\hline 0.52 & 0.862 & 0.959 & 21 & 17 \\
0.50 & 0.903 & 0.965 & 9.8 & 7.8 \\
0.49 & 0.923 & 0.968 & 6.7 & 5.3 \\
0.48 & 0.943 & 0.970 & 4.69 & 3.7 \\
0.47 & 0.963 & 0.972 & 3.35 & 2.7 \\
0.46 & 0.983 & 0.974 & 2.41 & 1.9 \\
0.45 & 1.004 & 0.975 & 1.75 & 1.4 \\
\hline
\end{tabular}

dence time will be relatively long. Since we aim for residence times shorter than $10 \mathrm{~s}$ the minimum temperature should be $T=755 \mathrm{~K}$ or higher.

Using the criteria we are able to calculate values of $U^{*} / \Theta_{a d}$, the results are listed in Table 3 . We estimated $S_{P}$ using $S_{P 3}$, because $H_{Y}=1.77>1$.

From Table 3 we conclude that the required cooling capacity must be $U^{*} / \Theta_{\mathrm{ad}}>=18.1$. Since $U^{*}$ depends on both the reactor tube diameter and the reactor load we will calculate the maximum tube diameter for varying reactor loads. A method outlined by Westerterp et al. (1984b) was used. The results are listed in Table 4.

For a reactor load of $u_{\mathrm{o}} / \varepsilon=1.8 \mathrm{~m} / \mathrm{s}$ the temperature and conversion profiles of $X_{A}$ and $X_{P}$ were calculated. The plots are shown in Fig. 8. The optimal residence time is $7.1 \mathrm{~s}$, which leads to an optimal reactor length of $L=12.8 \mathrm{~m}$.

We checked the design for changes in the feed concentration $C_{A O}$. For some values of $\Theta_{a d}$ temperature trajectories and conversion profiles are shown in Fig. 9 (a) and (b). As can be seen runaway occurs for $\Theta_{\mathrm{ad}}=0.87$ which corresponds to a concentration of 1.15 mole \% benzene.

The reactor will be overheated with more than $400 \mathrm{~K}$, which for sure will result in damage. Again we see that the margin between runaway conditions $\left(\Theta_{\mathrm{ad}}\right.$ $=0.87$ ) and conditions required for maintaining the correct yield $\left(\Theta_{\mathrm{ad}}=0.63\right)$ is large. This was also demonstrated previously by Westerterp, Ptasinsky and Overtoom (Westerterp and Ptasinsky, 1984a, b; Westerterp et al., 1984a; Westerterp and Overtoom, 1985), who for multiple reactions showed that a good selectivity is lost easier than runaway occurs.

Table 2. Determination of the maximum allowable temperature $T_{\mathrm{ma}}$ for a required minimum yield of $48 \% . j_{P}=14.9, B=0.055, p=1.18, q=0.85$

\begin{tabular}{cccccc}
\hline $\begin{array}{l}T_{\text {mi }} \\
(\mathbf{K})\end{array}$ & $X_{P \max }\left(T_{\text {mi }}\right)$ & $X_{\text {Amax }}\left(T_{\text {mi }}\right)$ & $\begin{array}{c}T_{\text {ma }} \\
(\mathbf{K})\end{array}$ & $\begin{array}{c}T_{\text {ma }}-T_{\text {mi }} \\
(\mathbf{K})\end{array}$ & $D a_{\text {lp }}$ \\
\hline 746 & 0.511 & 0.962 & 797 & 51 & 15.0 \\
755 & 0.506 & 0.963 & 797 & 42 & 12.3 \\
763 & 0.501 & 0.965 & 797 & 34 & 10.2 \\
772 & 0.496 & 0.966 & 797 & 25 & 8.4 \\
780 & 0.491 & 0.967 & 797 & 17 & 7.0 \\
789 & 0.486 & 0.968 & 797 & 8.5 & 5.9 \\
797 & 0.481 & 0.969 & 797 & 0.0 & 4.9 \\
\hline
\end{tabular}

Table 3. Required values of $U^{*} / \Theta_{\mathrm{ad}}$ according to the three criteria given by eqs (33), (38) and (39) in order to achieve a desired yield of at least $48 \%$ at a residence time shorter than $10 \mathrm{~s}$

\begin{tabular}{cccccc}
\hline & & & \multicolumn{3}{c}{$U^{*} / \Theta_{\text {ad }}$ according to } \\
\cline { 3 - 6 }$\Theta_{\text {ma }}$ & $\Theta_{c}$ & $\Theta_{\text {ad }}$ & $\begin{array}{c}\text { Equation } \\
(33)\end{array}$ & $\begin{array}{c}\text { Equation } \\
(38)\end{array}$ & $\begin{array}{c}\text { Equation } \\
(39)\end{array}$ \\
\hline 0.946 & 0.892 & 0.63 & 19.3 & 18.1 & 18.2 \\
\hline
\end{tabular}




\section{CONCLUSIONS}

Based on the model presented here for multiplereaction networks in a cooled tubular reactor we may conclude that the criteria derived enable us to select

Table 4. Maximum tube diameter and heat transfer coefficient for several reactor loads which satisfy the criteria given by Table 3

\begin{tabular}{ccc}
$\begin{array}{c}u_{0} / \varepsilon \\
(\mathrm{m} / \mathrm{s})\end{array}$ & $\begin{array}{c}U \\
\left(\mathrm{~W} / \mathrm{m}^{2} \mathrm{~K}\right)\end{array}$ & $\begin{array}{c}d_{t, \max } \\
(\mathrm{m})\end{array}$ \\
\hline 0.6 & 82 & $2.0 \times 10^{-2}$ \\
1.2 & 115 & $2.8 \times 10^{-2}$ \\
1.8 & 146 & $3.5 \times 10^{-2}$ \\
2.4 & 175 & $4.2 \times 10^{-2}$ \\
\hline
\end{tabular}

practical values of the design and operating variables in order to achieve a desired yield. It should be mentioned that the method presented requires kinetic information about both undesired reactions and the desired reaction, and the required tube length, can be obtained by numerical integration only and that the method will work in case of first-order reactions only.

Also it was shown that for the scheme discussed in this paper it is not possible to design a safely operating reactor in which a network of reactions is carried out where $100 \%$ yield can be approached at will.

\section{NOTATION}

A pre-exponential factor, $\mathrm{m}^{3} /(\mathrm{kg} \mathrm{s})$

$B$ dimensionless kinetic factor, $=\left(A_{X} / A_{P}\right) \exp \left[j_{P}(1-q)\right]$
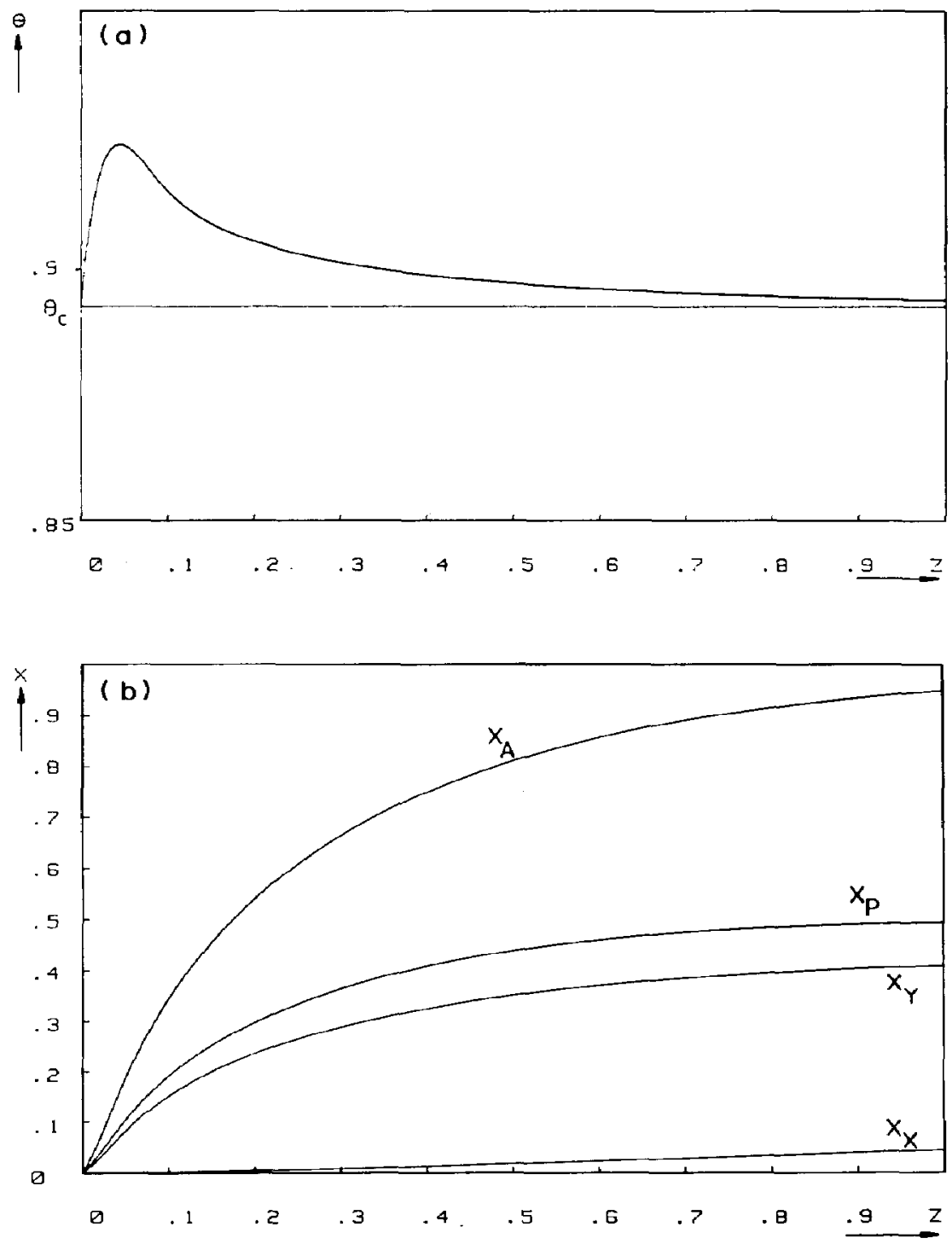

Fig. 8. Profiles for the temperature (a) and the conversion (b) for the design presented in the example. The oxidation of benzene to maleic anhydride. 

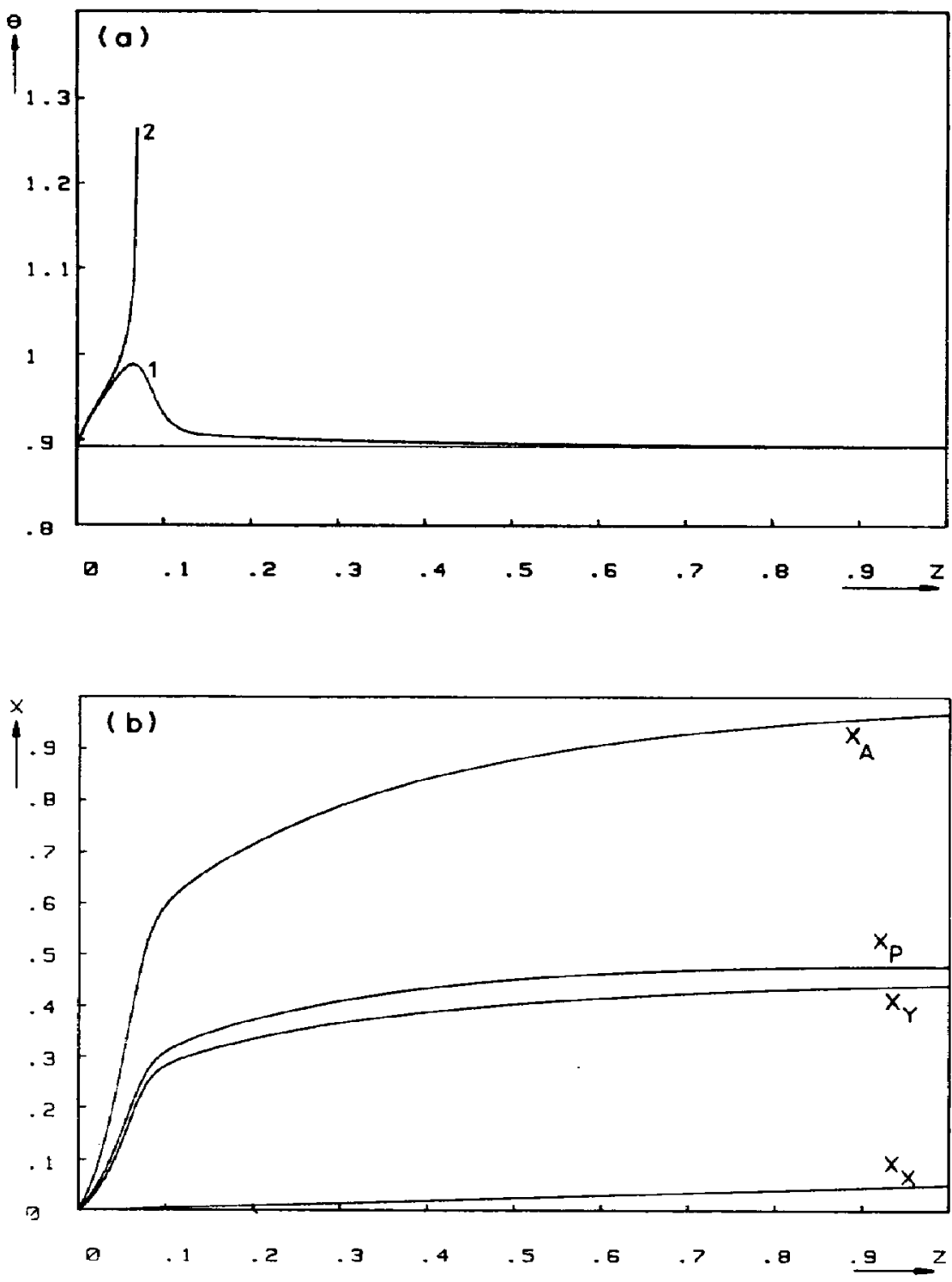

Fig. 9. Temperature (a) and conversion profiles (b) for changes in the feed concentration of the reactor designed in the example. For profile 1 in (a) and for the conversions in (b) the feed concentration is 1.12 mole \% benzene. For trajectory 2 in (a) the concentration is 1.15 mole \% benzene.

C concentration of species, $\mathrm{mol} / \mathrm{m}^{3}$

$C_{\mathrm{pg}} \quad$ specific heat of reaction mixture, $J /(\mathbf{k g ~ K})$

$d_{t}{ }^{\mathrm{pg}} \quad$ tube diameter, $m$

Da $\quad k_{R} \rho_{b} L / u$, dimensionless residence time

$E_{i} \quad$ activation energy for the reaction $i, J / m o l$

$H_{i} \quad \Delta H_{i} / \Delta H_{p}$, ratio of reaction heats

$(\Delta H)_{P}$ heat of reaction for the production of $P$ J/mol

$j_{P} \quad$ dimensionless

$=E_{P} /\left(R T_{R}\right)$

$k$ reaction rate constant, $\mathrm{m}^{3} /(\mathrm{kg} \mathrm{s})$

$K \quad k_{P} / k_{R}$, dimensionless rate constant

$k_{R} \quad$ reference reaction rate constant, $\mathrm{m}^{3} /(\mathrm{kg} \mathrm{s})$
$L \quad$ reactor length, $m$

p $\quad E_{Y} / E_{P}$

$q \quad E_{X} / E_{P}$

$\boldsymbol{R}_{w J} \quad$ rate of production of species $J, \mathrm{~mol} /(\mathrm{kg}$ cat s)

$S_{F} \quad$ integral selectivity achieved in the hot spot, $=\boldsymbol{X}_{\boldsymbol{P}} / \boldsymbol{X}_{\boldsymbol{A}}$

differential selectivity, $=\mathrm{d} X_{p} / \mathrm{d} X_{A}$ temperature, $K$

reference temperature, $K$

superficial gas velocity based on empty crosssection, $\mathrm{m} / \mathrm{s}$

total heat transfer coefficient, $W /\left(\mathrm{m}^{2} \mathrm{~K}\right)$ 
$\boldsymbol{X}$ relative degree of conversion

$z \quad$ coordinate in direction of flow, $m$

$Z \quad z / L$, dimensionless reactor length

\section{Greek letters}

$\varepsilon \quad$ bed porosity

$\rho_{b} \quad$ bulk catalyst density per unit of reactor volume, $\mathrm{kg} / \mathrm{m}^{3}$

$\rho_{g} \quad$ density of the reaction mixture, $\mathrm{kg} / \mathrm{m}^{3}$

$\Theta \quad T / T_{R}$, dimensionless temperature

$\Theta_{\mathrm{ad}} \quad-\Delta H_{P} C_{A d} / T_{R} \rho_{g} C_{\mathrm{pg}}$, dimensionless adiabatic temperature rise

\section{Subscripts}

A reactant

c coolant

hs hot spot

il lower point of intersection

iu upper point of intersection

lp longest possible

ma maximum allowable

max optimum under isothermal conditions

mi minimum required

o inlet conditions

opt absolute optimum

$P \quad$ product

sp shortest possible

$\boldsymbol{X}$ undesired product formed by the consecutive reaction

$Y$ undesired product formed by the parallel reaction

\section{REFERENCES}

Westerterp, K. R. and Overtoom, R. R. M., 1985, Safe design of cooled tubular reactors for exothermic multiple reactions; consecutive reactions. Chem. Engng Sci. 40, 155.

Westerterp, K. R. and Ptasinsky, K. J., 1984a, Safe design of cooled tubular reactors for exothermic multiple reactions; parallel reactions. Development of criteria. Chem. Engng Sci. 39, 235.
Westerterp, K. R. and Ptasinsky, K. J., 1984b, Safe design of cooled tubular reactors for exothermic multiple reactions; parallel reactions. The design and operation of an ethylene oxide reactor. Chem. Engng Sci. 39, 245.

Westerterp, K. R., Ptasinsky, K. J. and Overtoom, R. R. M., $1984 a$, Safe design of cooled tubular reactors for exothermic multiple first order reactions. ACS Symp. Ser. no. $237,323-335$.

Westerterp, K. R., Swaaij, W. P. M. van and Beenackers, 1984b, Chemical Reactor Design and Operation, pp. 305-307. John Wiley, Chichester.

Westerterp, K. R. and Westerink, E. J., 1988, Safe design of tank reactors for multiple reaction networks: uniqueness and multiplicity. To be published in Chem. Engng Sci.

Wohlfahrt, K. and Emig, G., 1980, Compare maleic anhydride routes. Hydrocarbon Processing 59, 83.

\section{APPENDIX}

Introduction to multiple hot spots

In previous studies criteria were developed that were based on the assumption that only one hot spot occurs. These criteria ensure that this hot spot temperature is not surpassed at any point in the reactor.

In the case when multiple reactions occur one may expect the possibility of more than one hot spot. For example two hot spots may occur in the case when two exothermic consecutive reactions are carried out: the first hot spot will be caused by the first reaction, and the second one will occur due to the second reaction.

The number and location of all hot spots that could possibly occur in the case when multiple reactions are carried out should be studied. We will use the reaction scheme presented as scheme (1) and discuss also the systems with either parallel or consecutive reactions, only putting the appropriate terms equal to zero.

\section{Features of multiple hot spots}

For the case that two hot spots occur, some possible trajectories are given in Figs $A^{-1}$ and $A 2$. We can easily see that between two hot spots a minimum must occur. In this minimum, of course, $d \Theta / d Z=0$ as was the case for the maxima. So we cannot discriminate between maxima and minima by the first derivative only. The extremum can be characterized by the second derivative of the temperature $\Theta$ with respect to the reactor length coordinate $Z$. For $d^{2} \Theta / d Z^{2}>0$ the extremum is a minimum, for $d^{2} \Theta / d Z^{2}<0$



Fig. A1. A temperature trajectory with two hot spots along the reactor tube. 


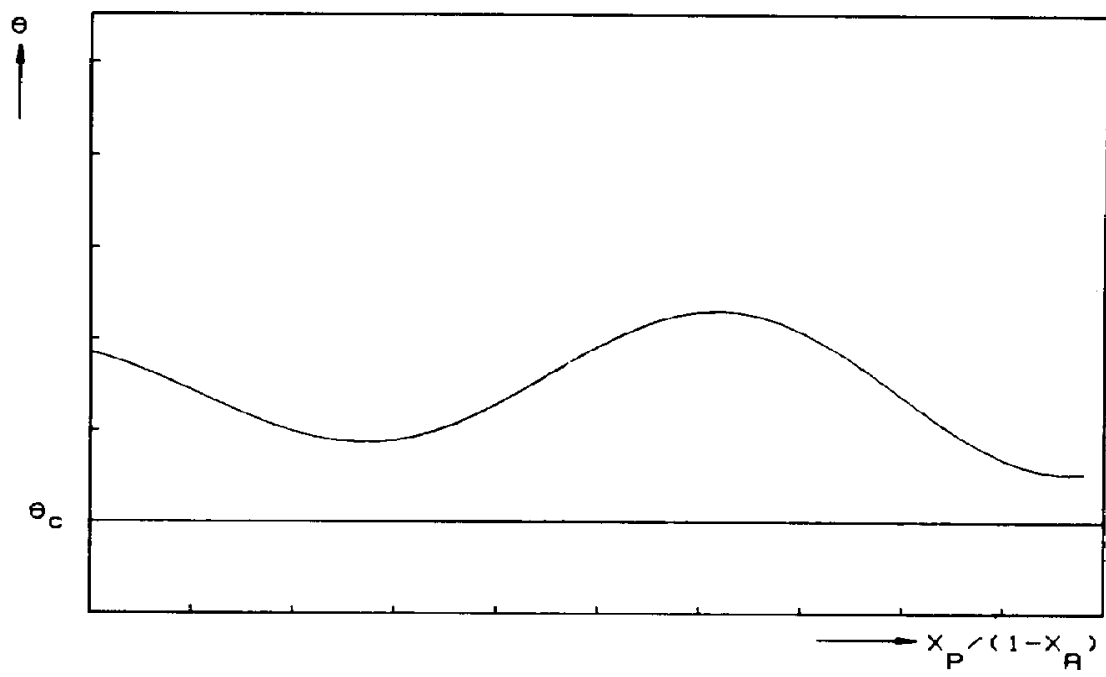

Fig. A2. A temperature trajectory with two hot spots of which the first one is located at the inlet.

it is a maximum. We will use the second derivative to define the border between regions where maxima and minima occur.

\section{Model equations}

In a tubular reactor which can be described by the pseudohomogeneous one-dimensional model, the temperature trajectories are fully determined by the following set of differential equations for the reaction system under consideration:

$$
\begin{aligned}
\frac{\mathrm{d} X_{A}}{\mathrm{~d} Z}= & D a\left(K+K^{P}\right)\left(1-X_{A}\right) \\
\frac{\mathrm{d} X_{P}}{\mathrm{~d} Z}= & D a\left[K\left(1-X_{A}\right)-B K^{q} X_{P}\right] \\
\frac{\mathrm{d} \Theta}{\mathrm{d} Z}= & D a \Theta_{\mathrm{ad}}\left[\left(K+H_{Y} K^{p}\right)\left(1-X_{A}\right)\right. \\
& \left.+H_{X} B K^{a} X_{P}\right]-D a U^{*}\left(\Theta-\Theta_{c}\right) .
\end{aligned}
$$

The locus of extrema, defined by $\mathrm{d} \Theta / \mathrm{d} Z=0$ is given by

$K_{\mathrm{ex}}+H_{Y} K_{\mathrm{ex}}^{p}+H_{X} B K_{\mathrm{ex}}^{q}\left(\frac{X_{P}}{1-X_{A}}\right)-\frac{U^{*}\left(\Theta_{\mathrm{ex}}-\Theta_{c}\right)}{\Theta_{\mathrm{ad}}\left(1-X_{A}\right)}=0$.

Instead of studying this locus curve in a three-parameter space $\left(\Theta, X_{A}, X_{P}\right)$ we will consider the conversion $X_{A}$ in terms of $X_{P} /\left(1-X_{A}\right)$ and study the relation in a $X_{P} /\left(1-X_{A}\right)$, $\Theta$ plane. The term $\hat{X}_{P} /\left(1-X_{A}\right)$ was chosen because this term also occurs in the relation used to characterize the extrema. It was demonstrated already before that the conversion $X_{A}$ may be replaced by the term $X_{P} /\left(1-X_{A}\right)$ within a certain range of differential selectivities.

The locus of extrema

We will use the isothermal reactor operating at the temperature in the extremum $\Theta_{\text {ex }}$ in order to obtain estimates of the values of $X_{A}$ in the extremum. The isothermal reactor is described by

$$
\frac{X_{P}}{1-X_{A}}=\frac{K}{K+K^{p}-B K^{q}}\left[\left(1-X_{A}\right)^{\frac{B K^{q}}{K+K^{p}-1}}-\left(1-X_{A}\right)\right] .
$$

After division by $1-X_{A}$ and some rearrangements this leads to

$$
\left(-\frac{X_{P}}{1-X_{A}} \cdot \frac{K+K^{p}-B K^{q}}{K}+1\right)^{\frac{1}{K+K^{q}}-1}=1-X_{A}
$$

Substitution of the value of $1-X_{A}$ of eq. (e) into eq. (d) leads to a relation for the temperature in the extremum:

$$
\begin{aligned}
K+H_{Y} K^{p}+H_{X} B K^{q} \frac{X_{P}}{1-X_{A}}=\frac{U^{*}}{\Theta_{\mathrm{ad}}}\left(\Theta-\Theta_{c}\right) \\
\times\left[1+\frac{X_{P}}{1-X_{A}}\left(\frac{K+K^{p}-B K^{q}}{K}\right)\right]^{\frac{1}{B-\frac{B K^{q}}{K+K^{*}}}}
\end{aligned}
$$

or after rearranging:

$$
\begin{gathered}
{\left[\frac{K+H_{Y^{\prime}} K^{p}+H_{X} B K^{q}\left(\frac{X_{P}}{1-X_{A}}\right)}{\frac{U^{*}}{\Theta_{\mathrm{ad}}}\left(\Theta-\Theta_{c}\right)}\right]^{1-\frac{B K^{q}}{K+K^{p}}}} \\
=1+\left(\frac{X_{P}}{1-X_{A}}\right)\left(\frac{K+K^{p}-B K^{q}}{K}\right)
\end{gathered}
$$

For known values of $U^{*} / \Theta_{\text {ad }}$ and $\Theta_{c}$ eq. (f) gives us the relation between $\Theta$ and $X_{P} /\left(1-X_{A}\right)$ in the extrema, both for the minima and the maxima. Some of these extrema curves are plotted in Figs A3 and A4 (curves $c, c_{r}$ and $c_{1}$ ). Inside the extrema curve the derivative $d \Theta / d Z$ is negative so the trajectories decrease, while outside the curve the derivative is positive; hence there the trajectories will increase. We can see that for diminishing values of $U^{*} / \Theta_{\text {ad }}$ the curve of extrema separates into two branches (Fig. A4, curves $c_{1}$ and $c_{r}$ ). Inside these branches $d \Theta / d Z$ is still negative so the trajectories will decrease in the inside region. In Figs A3 and A4 two trajectories are plotted for some values of $U^{*}$ and $\Theta$. keeping the ratio $U^{*} / \Theta_{\text {ad }}$ constant in each figure. In Fig. A4 the danger of more than one hot spot is clearly illustrated by trajectory a, where the temperature decreases at the onset of the reaction, but eventually a runaway occurs. For even smaller values of $U^{*} / \Theta_{a d}$ the left branch completely disappears.

\section{Characterization of all points on the locus of extrema}

The second derivative of $\Theta$ with respect to $\mathcal{Z}$ will be used to characterize the extrema defined by relation (f). The second 


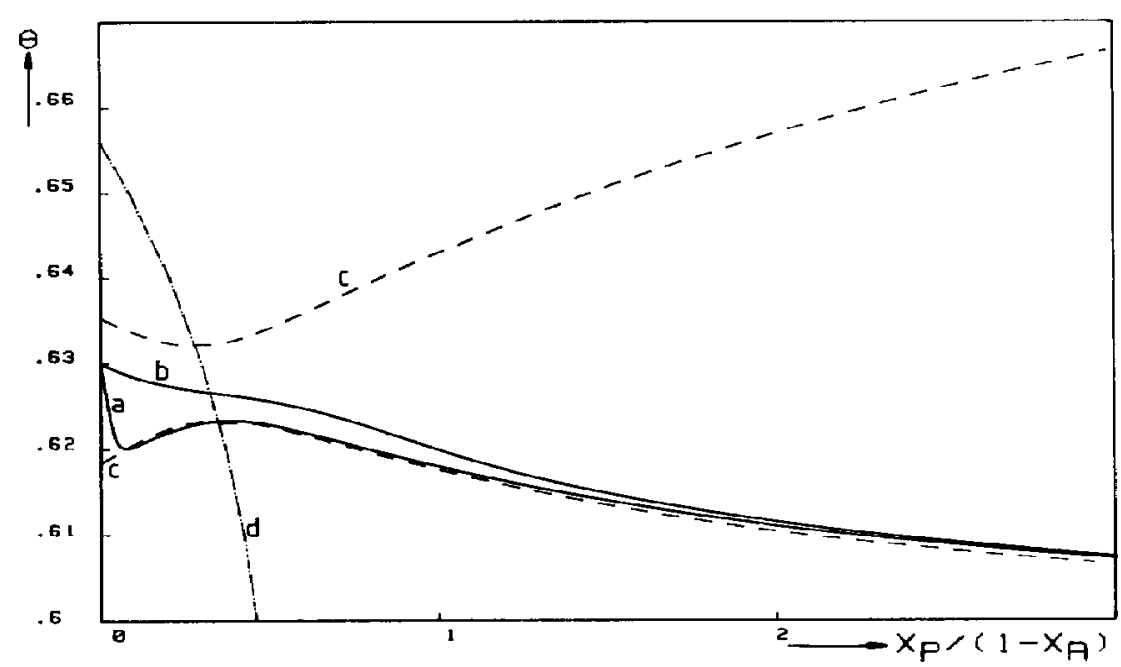

Fig. A3. Two branches of a locus of extrema curve (c), the curve (d) that marks the border between maxima and minima, and two trajectories (a) and (b). Data are $p=1.5, q=0.8, B=0.11, j_{P}=15, H_{X}=H_{Y}=2, \Theta_{c}$ $=0.6, \Theta_{0}=0.63$ and $U^{*} / \Theta_{\mathrm{ad}}=0.0053$. Trajectory (a) $\Theta_{\mathrm{ad}}=5, U^{*}=0.0265 ;$ (b) $\Theta_{\mathrm{ad}}=0.5, U^{*}=0.00265$.



Fig. A4. The locus of extrema curve with two branches ( $c_{r}$ and $c_{1}$ ), the border curve (d) and two trajectories (a) and (b). Same data as for Fig. A3, except now $U^{*} / \Theta_{\text {ad }}=0.00518$. Trajectory (a) $\Theta_{\text {ad }}=0.5, U^{*}=0.00259 ;$ (b) $\Theta_{\mathrm{ad}}=5, U^{*}=0.0259$.

derivative as derived from eq. (c) is

$$
\begin{aligned}
\frac{1}{D a} \cdot \frac{\mathrm{d}^{2} \Theta}{\mathrm{d} Z^{2}}= & \Theta_{\mathrm{ad}}\left[\left(1+p H_{Y} K^{p-1}\right)\left(1-X_{A}\right)\right. \\
& \left.+q H_{X} B K^{q-1}\right]\left(\frac{\mathrm{d} K}{\mathrm{~d} \Theta}\right)\left(\frac{\mathrm{d} \Theta}{\mathrm{d} Z}\right) \\
& +\Theta_{\mathrm{ad}}\left[-\left(K+H_{Y} K^{p}\right)\left(\frac{\mathrm{d} X_{A}}{\mathrm{~d} Z}\right)\right. \\
& \left.+H_{X} B K^{q}\left(\frac{\mathrm{d} X_{P}}{\mathrm{~d} Z}\right)\right]-U^{*}\left(\frac{\mathrm{d} \Theta}{\mathrm{d} Z}\right)
\end{aligned}
$$

Since only the sign of the second derivative in the extremum is of interest and furthermore in the extremum $\mathrm{d} \Theta / \mathrm{d} Z=0$ holds this relation can be rearranged to

$$
\begin{aligned}
\left.\frac{1}{D a} \cdot \frac{\mathrm{d}^{2} \Theta}{\mathrm{d} Z^{2}}\right|_{Z_{\mathrm{ex}}} & \Theta_{\mathrm{ad}}\left[-\left(K+H_{Y} K^{p}\right)\left(\frac{\mathrm{d} X_{A}}{\mathrm{~d} Z}\right)\right. \\
& \left.+H_{X} B K^{q}\left(\frac{\mathrm{d} X_{P}}{\mathrm{~d} Z}\right)\right] .
\end{aligned}
$$

From this equation we can easily see that for parallel reactions with $B K^{4}=0$ we have only maxima since $d^{2} \Theta / d Z^{2}$ is always negative. As no minimum occurs there can be one hot spot only for parallel reactions. 
Substitution of eqs (a) and (b) into eq. (g) leads to

$$
\begin{aligned}
\frac{-1\left(\frac{\mathrm{d}^{2} \Theta}{\mathrm{d} Z^{2}}\right)}{D a^{2} \Theta_{\mathrm{ad}}\left(1-X_{A}\right)}= & \left(K+H_{Y^{\prime}} K^{p}\right)\left(K+K^{P}\right) \\
& -H_{X} B K^{a}\left(K-B K^{a} \cdot \frac{X_{P}}{1-X_{A}}\right) .
\end{aligned}
$$

The right-hand side of this equation gives a relation that can be used to characterize the extrema: if the right-hand side of relation (h) is negative the extremum is a minimum, and vice versa. The borderlines between regions where minima and maxima occur is obtained if the right-hand side of eq. (h) is set equal to zero, so that

$$
\left(K+H_{Y^{\prime}} K^{p}\right)\left(K+K^{p}\right)-H_{X} B K^{q}\left[K-B K^{q}\left(\frac{X_{P}}{1-X_{A}}\right)\right]=0
$$

which leads to the following expression for the borderline curve:

$$
\left(\frac{X_{P}}{1-X_{A}}\right)=\frac{K}{B K^{4}}-\frac{\left(K+H_{Y} K^{p}\right)\left(K+K^{p}\right)}{H_{X} B^{2} K^{2 q}} .
$$

Typical plots of relation (i) are given in Figs A3 and A4 as curves $d$. The second derivative is positive at the left of the curve $d$ so that the extrema on the locus of extrema curve are minima while on the right-hand side of the curve the extrema are maxima. In Fig. A 3 the area enclosed by curve $c$ is still continuous but already exhibits a constriction. At still lower values of $U^{*} / \Theta_{\mathrm{ad}}$ it falls apart in two separate areas, whereas curve $d$ now lies between these areas.

\section{The existence of minima in temperat ure trajectories}

The locus of extrema and the regions of minima and maxima can be used to investigate the existence of temperature minima. To reach a minimum the temperature must decrease. For a trajectory to decrease it must be situated inside the region indicated by the locus of extrema curve. After the minimum has been passed the temperature should rise again. From the plots in Figs A3 and A4 where the horizontal axis equals $\Theta_{c}$ we can easily see that only for the inlet condition $\Theta_{o}>\Theta_{r}$ can we have a trajectory that decreases at the onset of the reaction, as shown in Fig. A2. It is not possible to obtain trajectories as presented in Fig. Al since in that case the temperature should rise inside the locus of extrema branch.
Discussion and conclusions

Apparently it is possible to obtain more than one hot spot in the case of a multiple-reaction system like the one presented. We can easily understand that systems with parallel reactions only can have only one hot spot since in that case the reactant has to make a choice between either one of the reactions. In the case of consecutive reactions intrinsically the reactant fed can produce heat twice: first in the reaction $A \rightarrow P$ and afterwards again in $P \rightarrow X$. As the reaction proceeds the concentration of A diminishes continuously. So from the concentration point of view the first reaction cannot be ignited again. However the concentration of $\mathbf{P}$ continuously increases. This also increases the reaction rate of $P \rightarrow X$ and if the combined conditions of concentration and temperature are favourable a second ignition can occur.

The reasoning given above holds too for the reaction system consisting of both parallel and consecutive reactions. Based upon the discussions above we may conclude that more than one hot spot may occur in the case of a reaction system like the one presented in a tubular reactor.

We can understand that no multiple hot spot can occur in case the inlet temperature $\Theta_{o}$ and the cooling temperature $\Theta_{c}$ are higher than the point of intersection of the borderline (d) with the $X_{P} /\left(1-X_{A}\right)=0$ axis. The point of intersection between curvedd and the $X_{P} /\left(1 \cdots X_{A}\right)=0$ axis is given by

$$
\frac{X_{P}}{1-X_{A}}=\frac{K}{B K^{q}}-\frac{\left(K+H_{Y} K^{p}\right)\left(K+K^{p}\right)}{H_{X} B^{2} K^{2 q}}=0 .
$$

For the inlet conditions $\Theta_{o}=\Theta_{c}$ to be above the point of intersection the following criterion must be satisfied:

$$
\frac{K}{B K^{q}}-\frac{\left(K+H_{Y} K^{p}\right)\left(K+K^{p}\right)}{H_{X} B^{2} K^{2 q}}<0 \text { at } \Theta=\Theta_{c}
$$

or after some rearrangements:

$$
\frac{H_{X} B K_{c}^{q+1}}{\left(K_{c}+H_{Y} K_{s}^{P}\right)\left(K_{c}+K_{c}^{P}\right)}<1 .
$$

In the case of our example the critical value of $\Theta_{c}$ for which multiple hot spots may occur is found by setting eq. (j) equal to zero and solving it for $\Theta_{c, c r i t}$. This leads to

$$
\Theta_{c, c r i t}=0.40
$$

which corresponds to a value of $66^{\circ} \mathrm{C}$. Duc to the low value of this temperature which would lead to very long residence times we may conclude that under practical conditions no multiple hot spots will occur in the reactor of our example. 